\title{
Development of a compliant legged quadruped robot
}

\author{
M M GOR ${ }^{1, *}$, P M PATHAK ${ }^{1}$, A K SAMANTARAY $^{2}, \mathrm{~K}$ ALAM $^{1}, \mathrm{P} \mathrm{KUMAR}^{1}, \mathrm{D}$ ANAND $^{1}$, \\ $\mathrm{P}_{\text {VIJAY }}{ }^{1}, \mathrm{R}_{\text {SARKAR }}{ }^{1}, \mathrm{~J}_{-M} \mathrm{YANG}^{3}$ and $\mathrm{S}$ W KWAK ${ }^{4}$ \\ ${ }^{1}$ Indian Institute of Technology Roorkee, Roorkee, India \\ ${ }^{2}$ Indian Institute of Technology Kharagpur, Kharagpur, India \\ ${ }^{3}$ Kyungpook National University, Daegu, South Korea \\ ${ }^{4}$ Keimyung University, Daegu, South Korea \\ e-mail: mehulmgor@gmail.com; pushpfme@iitr.ac.in; samantaray@mech.iitkgp.ernet.in; \\ kamran.ec.iitr@gmail.com; paiyusah@gmail.com; divye2011@gmail.com; pvijay497@gmail.com; \\ rohaneeiitr@gmail.com; jmyang@ee.knu.ac.kr; ksw@kmu.ac.kr
}

MS received 19 May 2016; revised 11 November 2017; accepted 1 June 2018; published online 18 June 2018

\begin{abstract}
This paper presents the detailed steps for design and development of a compliant legged fault tolerant quadruped robot where each leg has two links and two motorized revolute joints for locomotion. The body and upper links of legs are rigid whereas the lower link of each leg is compliant. Amble gait is demonstrated on the developed robot. Safety and reliability are the most critical issues for the quadruped robot. During the failure of any joint, performance of quadruped robot is affected. In this paper, locked joint failure is also discussed. Strategies for fault tolerant control of the quadruped are developed and experimentally validated. The developed robot can be used for various hardware-in-the-loop controller prototyping such as reconfiguration, fault tolerant control, and posture control, etc. pertaining to quadruped robots.
\end{abstract}

Keywords. Quadruped robot; compliant leg; locked joint failure.

\section{Introduction}

Quadruped robots have many practical advantages over other mobile robots. However, development and operation of working model of a quadruped robot is not easy. More and more quadrupeds are developed now-a-days. Some of them are Baby Elephant [1], Cheetah-cub [2], HyQ [3], LittelDog [4, 5] and Tekken [6, 7]. Main intensions behind their development were to explore their use in hazardous and unstructured environments.

Hydraulically actuated Baby Elephant [1] was designed to work as mechanical carrier. It has compliant legs and 12 degrees-of-freedom (DOFs). In [1], multi-body dynamic simulation software was used to compare experimental results with simulations. Electrically actuated Cheetah-cub [2] was designed for fast locomotion. Cheetah-cub's leg has spring loaded pantograph mechanism with multiple segments. Robot's self-stabilizing properties was demonstrated in hardware model and in simulation carried out in Webots software. HyQ [3] has 12 DOFs and it was designed to perform highly dynamic tasks like jumping and running. It has both the hydraulic and electric actuation system. During running and jumping, generated impact forces were absorbed by hydraulic actuation mounted on hip and knee

*For correspondence joints in the flexion/extension plane of the leg. The hip abduction/adduction joint was actuated by brushless electric motor which provides constant output torque. LittleDog $[4,5]$ has 12 DOFs and each joint is operated by high gain servo motors. Sensors mounted on the robot measure body orientation, joint angles and ground-foot contact. Sensing, communication and actuators are controlled by an onboard computer. Tekken $[6,7]$ is a light weight $(4.3 \mathrm{~kg})$ manually operated compliant legged quadruped robot. It has $16 \mathrm{DOFs}$ with 3 joints around pitch axis (ankle, knee and hip) and 1 hip joint around yaw axis at each leg.

Locked joint failure is a kinematic failure under which a joint cannot move and is locked in place. However, body supporting ability is preserved. Due to this characteristic feature, the failed leg still contributes partially towards the robot locomotion. The locked joint failure is very common and occurs due to friction in the bearings, slipping of clutches, broken gear teeth jamming the motor, and misalignment/bending on the rotor shaft. In this situation, locomotion can be carried out by either implementing fault tolerant gait pattern or applying control laws to the redundant hardware. Different fault tolerant gaits have been suggested by Lee [8] and Yang [9-11] for locked joint failures. Fault accommodation through reconfiguration is accomplished by on-the-fly deployment/activation of alternate standby devices, called hardware redundancies, in 


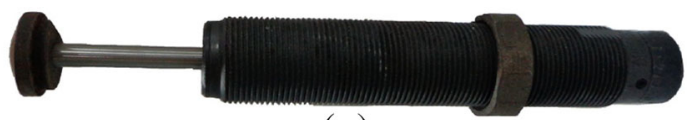

(a)

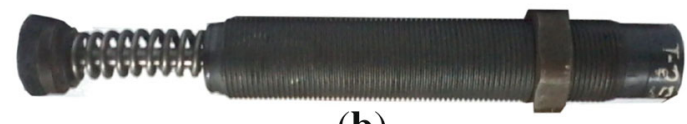

(b)

Figure 1. Compliant link: (a) without spring, (b) with spring.
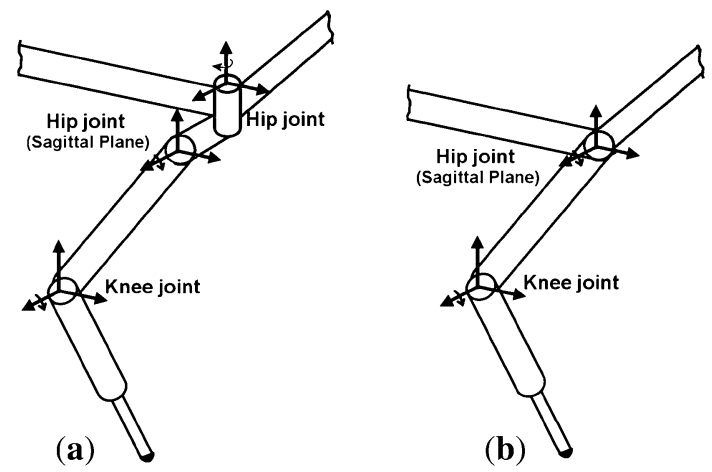

Figure 2. (a) Common kinematic structure of quadruped, (b) kinematic structure of developed quadruped.

the place of faulty actuators and adapting control laws to the modified system's architecture [12].

This paper introduces the quadruped robot, developed as a part of Indo-Korea joint research project at Robotics and Control Lab of Indian Institute of Technology Roorkee, India. It is an electrically actuated compliant legged quadruped robot with two DOFs per leg. Main objective behind this development is to explore untouched research areas in quadruped robots such as reconfiguration, fault tolerant control, posture control, etc. The design is based on extensive modeling and simulation studies published earlier [13-15]. In this paper, fault tolerant control through reconfiguration is proposed. A novel robot-on-robot type moving appendage, which is realized in the form of a two DOF robot with a payload at its end, is proposed. This appendage device may serve as a redundant hardware, which is to be activated only when the base quadruped robot experiences a locked joint failure.

This paper is organized as follows. In section 2, the entire process of development of the quadruped robot is presented. It covers details about the compliant link, kinematic structure, joint torque estimation, actuation system, controllers and the fabrication process. Section 2 also presents the experimental results when the robot moves in amble gait. In section 3, fault tolerant control through reconfiguration by using hardware redundancy is discussed. This is further validated through simulation and experimental results. Finally, section 4 provides conclusions and draws perspectives.

\section{Development of quadruped robot}

This section discusses the quadruped specification and design studies. As per our design objective, the quadruped should be able to walk with different static and dynamic gait patterns. At the initial stage it is difficult to decide the physical specification. Design process was initiated from the commercially available components size and specifications. As the robot is compliant legged, we start from a commercially available compliant link.

\subsection{Compliant link}

As natural evolution, humans and animals have legs with spring like behavior that enables them to absorb shock on impact, maintain stability, and also to initiate sudden motion. Generally, mechanical springs are used to introduce compliance in the structure of robot $[7,16]$. Compliance in the link improves locomotion of quadruped robot. But over compliance reduces locomotion speed and increases posture disturbance. So, optimizing the compliance is required for a specific robot configuration. Such a design is facilitated by simulating the model of quadruped $[13,14]$ with some initial assumptions of link and body parameters and then evolving the design through parameter variations. Number of simulations can be carried out by varying the stiffness and resistance, and its values can be finalized for maximum locomotion speed and minimum posture disturbance of robot. This optimum stiffness and load coming on each leg becomes the key parameters for designing the spring used in the compliant link. Number of turn of spring ' $n$ ' can be decided as,

$$
n=\frac{\delta G d}{8 W C^{3}}
$$

where, $\delta$ is axial deflection derived from $\operatorname{load}(W) /$ stiffness $(K), G$ is the modulus of rigidity of spring material, $d$ is wire diameter, $C$ is spring index derived from coil diameter $(D) /$ wire diameter $(d)$. The robot developed in this research uses a commercially available prismatic link as shown in figure 1(a). Internal hydraulic pressure provides damping and a spring is used to produce compliance in the link as shown in figure 1(b) with the above discussed design considerations.

\subsection{Kinematic structure}

The kinematic structure of a quadruped robot should be simple and yet allow the robot to perform a wide range of tasks. As shown in figure 2(a), the hip and knee joints in the 

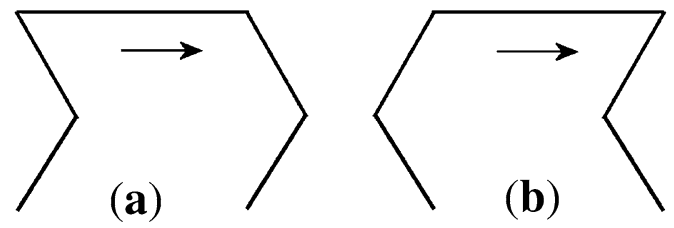

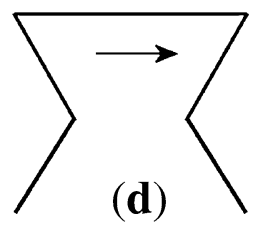

Figure 3. Leg configuration: (a) forwardforward, (b) backward-backward, (c) forward-backward, (d) backward-forward.

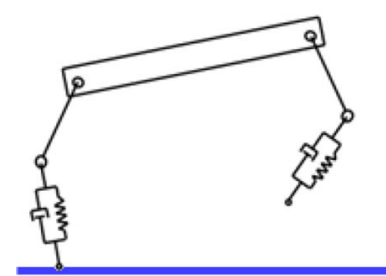

Back stance

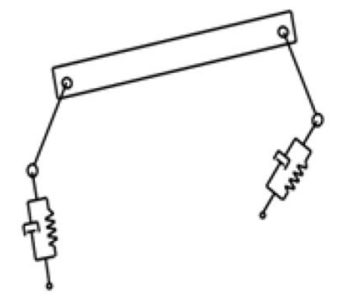

Flight after back stance

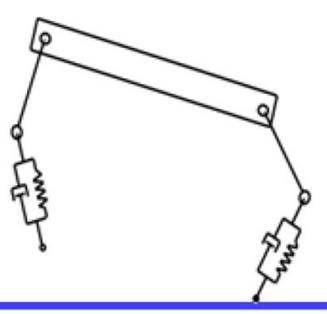

Front stance

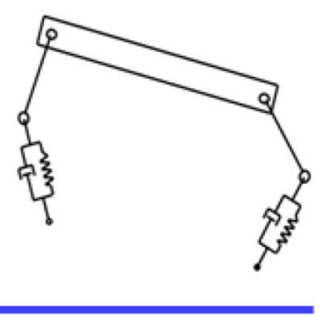

Flight after front stance

Figure 4. The four running states of bounding gait [15].

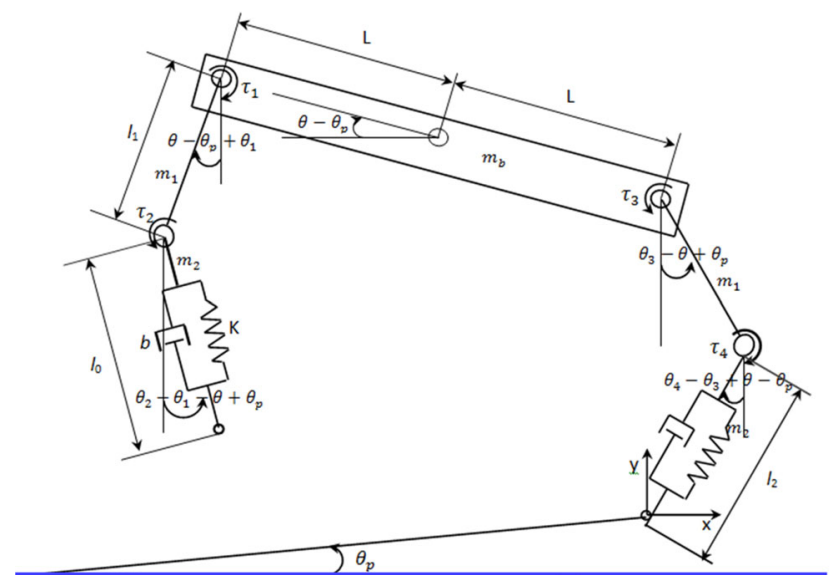

Figure 5. Sagittal plane model of quadruped [15].

sagittal plane and one hip joint on the vertical perpendicular plane is a common kinematic design structure. Thus, there are 3 DOFs per leg. However, as an initial attempt, the robot is constructed with 2 DOFs per leg whose corresponding kinematic structure is shown in figure 2(b). Provision is kept in the design to include the third degree of freedom in the future.

Legs may be attached with the body in different ways as shown in figure 3. If knee joint points to the front of the robot then it is termed as forward configuration and if it points to the back of the robot then it is termed as backward configuration. Thus, four possible configurations are forward-forward, backward-backward, forward-backward and backward-forward. It is reported in literature [17] that backward-forward configuration is most beneficial one as it reduces slippage between feet and ground and improves the stability of motion. Therefore, backward-forward leg configuration is adopted in this research.

\subsection{Joint torque estimation}

Before finalizing size and type of actuation system it is necessary to estimate the joint torque that is required for desired tasks and the joint motors are to be selected based on the estimated torque demand on those. For torque estimation, a four-legged robot model with bounding gait presented in [15] is utilized. Its four phases, i.e., back stance, flight after back stance, front stance and flight after front stance which are shown in figure 4 [15].

Figure 5 shows sagittal plane model of quadruped robot [15] which is considered for the dynamic model development. All links and body are assumed to be slender and uniform. Nomenclatures used in this paper are listed at the end of paper. Torque at each joint is calculated by using the equations shown in "Appendix A". Movement starts from the initial position where it is assumed that each joint has position $0 \mathrm{rad}$, velocity $0 \mathrm{rad} / \mathrm{s}$ and acceleration $3 \mathrm{rad} / \mathrm{s}^{2}$. It is assumed that the robot has to move on a maximum of $0.25 \mathrm{rad}$ sloped surface. Torque values obtained for the said movement are $5.83 \mathrm{Nm}, 2.81 \mathrm{Nm}, 6.38 \mathrm{Nm}$ and $8.37 \mathrm{Nm}$ for joints 1, 2, 3 and 4, respectively. Estimating the higher side of torque is a safe part of the design process. Considering a factor of safety, the estimated torque is finalized as $15 \mathrm{Nm}$. Thus, actuation system must be able to produce $15 \mathrm{Nm}$ torque. 
Table 1. Major specifications of Maxon motor unit [23].

\begin{tabular}{lc}
\hline Parameter & Value \\
\hline Brushless EC-4 pole motor (Maxon) & \\
Nominal voltage & $48 \mathrm{~V}$ \\
No load speed & $16,500 \mathrm{rpm}$ \\
No load current & $422 \mathrm{~mA}$ \\
Nominal speed & $15,800 \mathrm{rpm}$ \\
Nominal torque (max. continuous torque) & $118 \mathrm{~m} \mathrm{Nm}$ \\
Stall torque & $3430 \mathrm{~m} \mathrm{Nm}$ \\
Max. efficiency & $89 \%$ \\
Encoder with Line Driver (Maxon) & \\
Counts per turn & 1000 \\
Number of channel & 3 \\
Planetary gearhead GP42C (Maxon) & \\
Reduction & $230: 1$ \\
Number of stages & 4 \\
Maximum continuous torque & $15 \mathrm{Nm}$ \\
\hline
\end{tabular}

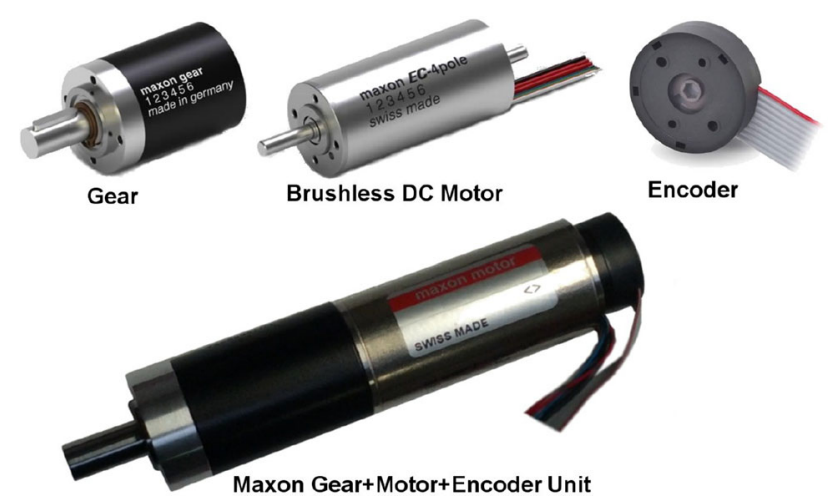

Figure 6. Electric actuation system for quadruped robot [23].

\subsection{Actuation system}

Hydraulic, pneumatic and electric actuations are usually used in the robots. Each actuation system has its own merits and demerits.

Quadruped robot developed by Raibert [18], Titan XI [19] and Baby Elephant [1] are actuated by hydraulic actuation system. HyQ [3] is also partially actuated by hydraulic system. Hydraulic actuation system generally offers high force. Due to low compressibility of fluid, hydraulic actuators also give a quick response. However, there are drawbacks of hydraulic actuation system such as oil leakages, change of oil viscosity with temperature, and non-linear characteristics of the hydraulic system leading to difficulty in control system development.

Pneumatic actuation is also a choice for quadruped robots. Hirose laboratory of the Tokyo Institute of Technology has developed a hybrid quadruped robot Airhopper
[20] in which each leg made up of four bar link which is actuated by three single acting pneumatic cylinders and a wheel attached at the leg tip is actuated by electric actuation. Pneumatic actuation system does not have the problem of oil leakage and oil flammability, and there is no need of return lines for the fluid. However pneumatic actuation is not the best choice for lower power-to-weight ratio, dynamic locomotion and quick response (due to compressibility of air).

Electric actuation system is most commonlyused in robots. Now-a-days, electric motors are available in big range of sizes and performance; they are more compact and economical. More and more quadrupeds are using electric actuation system. Some of them are Scout-II [21], Tekken II [7], KOLT [16] and Cheetah-cub [2]. In case of electric actuation system, compliance in the legs is realized with springs for energy efficient locomotion. Compliance in the legs also helps to protect the gear unit of electric actuator from excessive impact force.

In the present work, electric actuation system is used. Several kinds of electric motors are available and they are distinguished by current profile (AC/DC), number of coils, their configuration and type of synchronization. There are $\mathrm{AC}$ motors and brushed and brushless DC motors. It is found that brushless DC motors are most suitable for robotics application as they offer high speed, torque and efficiency, and low maintenance in comparison to other motors. Brushless DC motors have electronically controlled commutation systems. Due to absence of brushes, they are faster and more efficient because brushes create friction. Also, maintenance is less as there is no need to periodically replace brushes as in a brushed motor. However, brushless DC motors are costlier and their controllers are more complicated [22]. Looking at the positive side of brushless DC motors, brushless DC motors made by Maxon are used in this research. Motor unit comprising motor, gear head and encoder are chosen in such a way so that the entire unit can supply torque as estimated in previous section. Table 1 shows the major specifications of the Maxon motor and controller unit. Figure 6 shows Maxon motor, gear head and encoder parts separately and also as a combined unit.

\subsection{Controllers}

Controllers are the brain of the entire quadruped system. To maintain better compatibility with the actuation system, Maxon controller has been used. For the position control, a discrete PID controller with anti-windup, acceleration feed forward and velocity feed forward is implemented using a digital signal processor (DSP) where the sample time (TS) is taken as $1 \mathrm{~ms}$, which is much smaller than the mechanical time constant of a typical drive system. As per Maxon documentation, compatible controller for selected drive is EPOS2 $\mathrm{P} 24 / 5$, which is a freely programmable positioning 
Table 2. Major specification of Maxon controller unit [23].

\begin{tabular}{lc}
\hline Parameter & Value \\
\hline EPOS2 P 24/5 Controller (Maxon) & \\
Operating voltage & $11-24$ VDC \\
Digital inputs & 6 \\
Analog inputs & 2 \\
Digital outputs & 4 \\
Interface & RS232, CAN and USB 2.0 \\
\hline
\end{tabular}

controller [23]. Major specifications of this controller are shown in Table 2. To control eight motor units one need eight controllers. There is one master and seven slave controllers. Each controller controls one motor unit. Master controller does positioning control of its own motor as well as sends signal to other slave controllers to perform as per the program. Other characteristic of master controller EPOS2 $\mathrm{P}$ are IEC 61131-3 programmable, CANopen master function, multiple axis system via CAN Bus CANopen, point to point control unit and interpolated position mode. Master controller communication with computer, its own motor and other controller is shown in figure 7.

EPOS studio is used for programming according to IEC61131-3. Many decentralized controls can be easily managed with complex programs in EPOS studio. Desired input data are given in terms of encoder increment which is called quad count (qc). Entire process of locomotion planning and execution are presented in the flow chart as shown in figure 8.

\subsection{Sensors}

Information about body's angular and translational motion is obtained by MPU 6050 sensor. The InvenSense MPU-6050 [24] sensor contains a MEMS accelerometer and a MEMS gyro in a single chip. It is very accurate, as it contains 16-bits analog to digital conversion hardware for each channel. Therefore it captures the $\mathrm{x}, \mathrm{y}$ and $\mathrm{z}$ channels at the same time. This sensor is mounted on the top of the robot body. Over and above, a 3D motion capture system may also be used to determine position and velocity of leg tip. Commercially available PTI Visualeyez motion trackers [25] is used in this research because they are highly accurate and calibration free tracking system. These trackers are able to continuously collect information about the markers which are attached on the leg tip.

\subsection{Fabrication and final assembly}

After finalizing link, actuation system and controllers, a CAD model of the quadruped is prepared. Specifications of the developed quadruped robot are listed in table 3. Initially, as shown in figure 9, possible arrangements of the controller on the robot are evaluated. Finally, a cabinet is prepared to arrange all eight controllers on the quadruped as shown in figure 10. In the developed quadruped robot each leg has two electric motors per leg, but provision is also kept (as shown in the CAD model) to introduce third degree of freedom by fitting third motor at each leg. After satisfactory visualization of CAD model, fabrication of

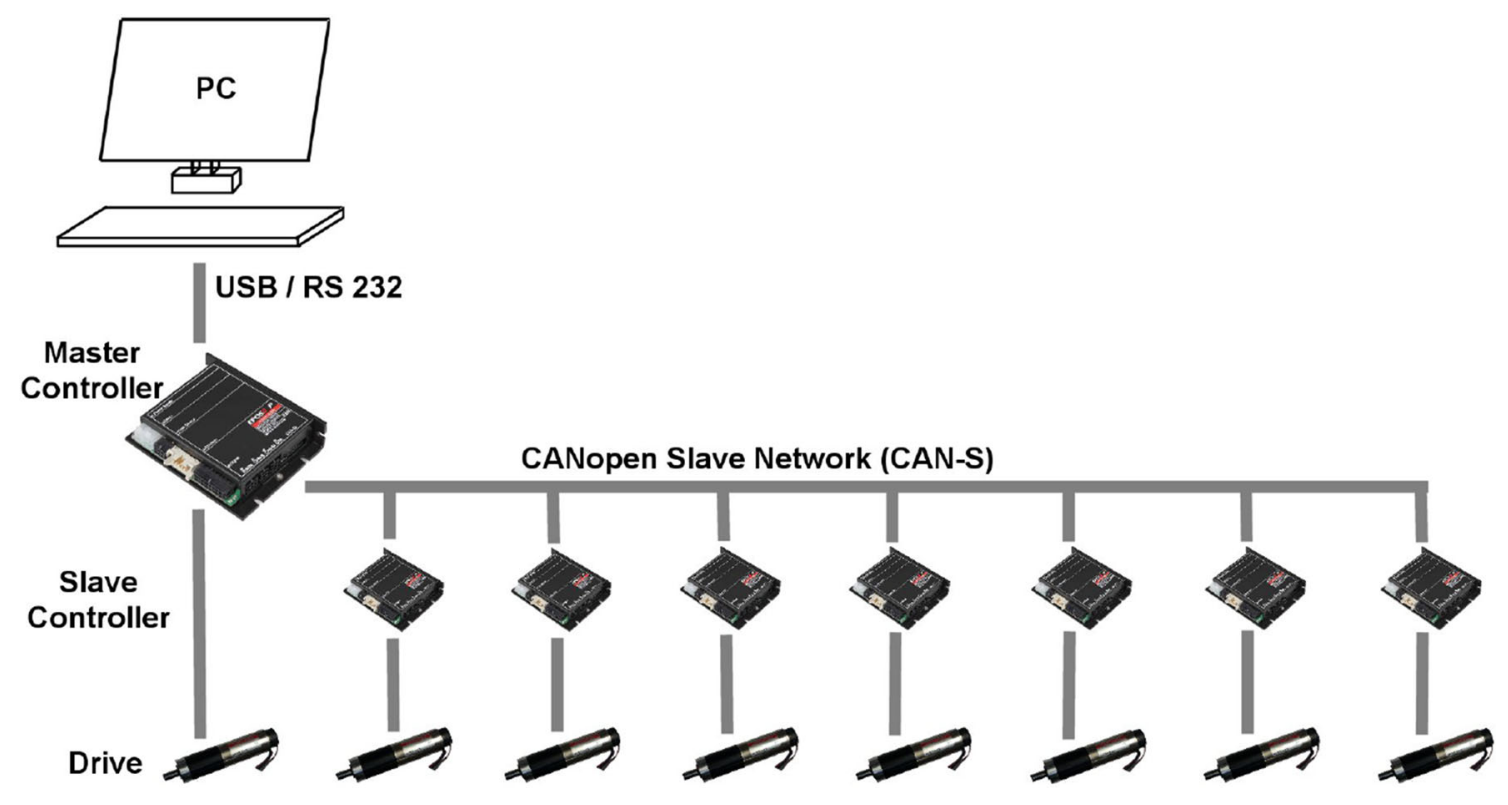

Figure 7. Controller connections with motors [23]. 


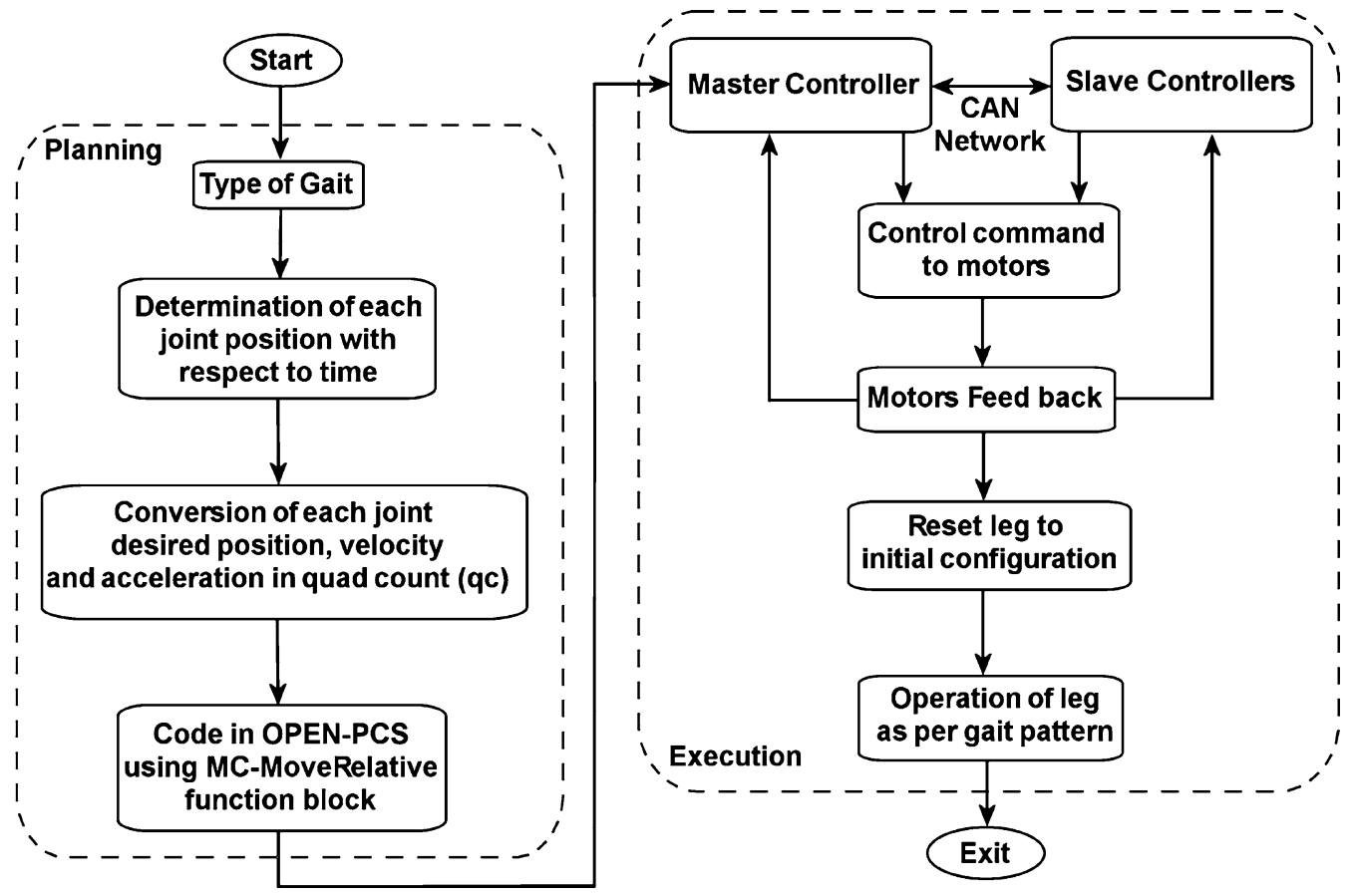

Figure 8. The flow chart of quadruped locomotion planning and execution.

Table 3. Specifications of quadruped robot developed at IIT Roorkee, India.

\begin{tabular}{lc}
\hline Parameter & Value \\
\hline Length of upper link & $0.225 \mathrm{~m}$ \\
Length of lower (compliant) link & $0.190 \mathrm{~m}$ \\
Body length & $0.500 \mathrm{~m}$ \\
Body width & $0.420 \mathrm{~m}$ \\
Body thickness & $0.065 \mathrm{~m}$ \\
Quadruped robot weight (approx.) & $15 \mathrm{~kg}$ \\
Main power source & $24 \mathrm{VDC}, 63$ \\
\hline
\end{tabular}

quadruped robot was started. Patterns were prepared for hub and upper link and they were casted from aluminum by sand casting process and then they were machined for final sizing. Robot chassis was prepared from aluminum channels. Lower link is ready made purchased and then it is modified by introducing the spring for leg compliance. The cabinet is fabricated from Galvanized Iron Sheet. Two stands are prepared from mild steel to hold robot while it is not moving. The final shape of the developed robot is shown in figure 11 .
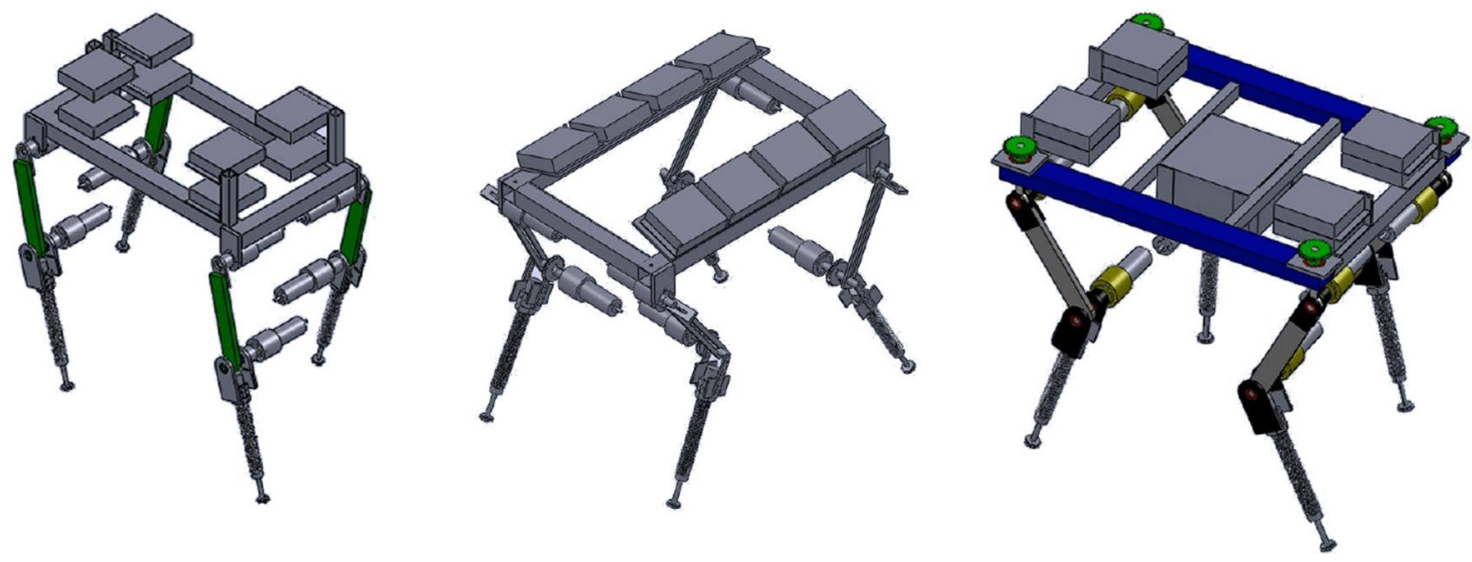

Figure 9. CAD models for possible arrangements of controllers on quadruped robot. 


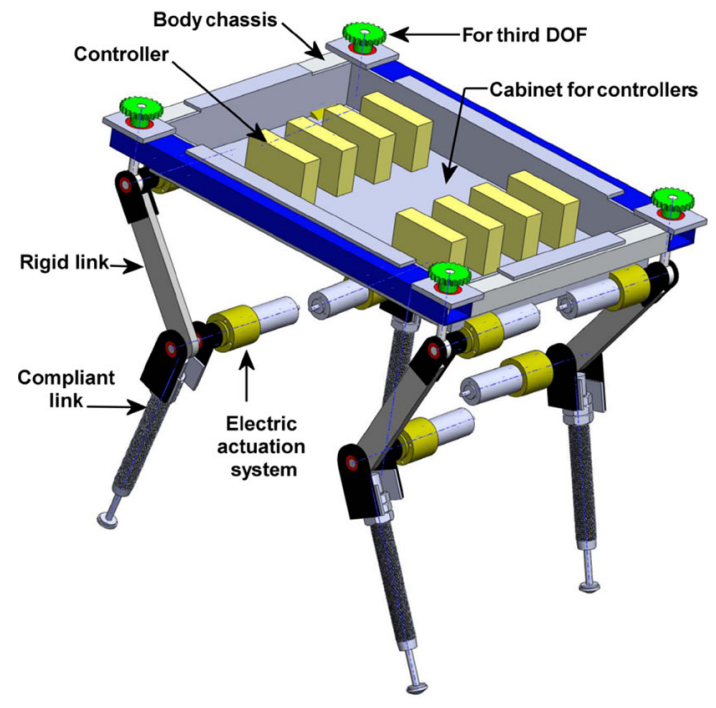

Figure 10. Solid model of quadruped robot.

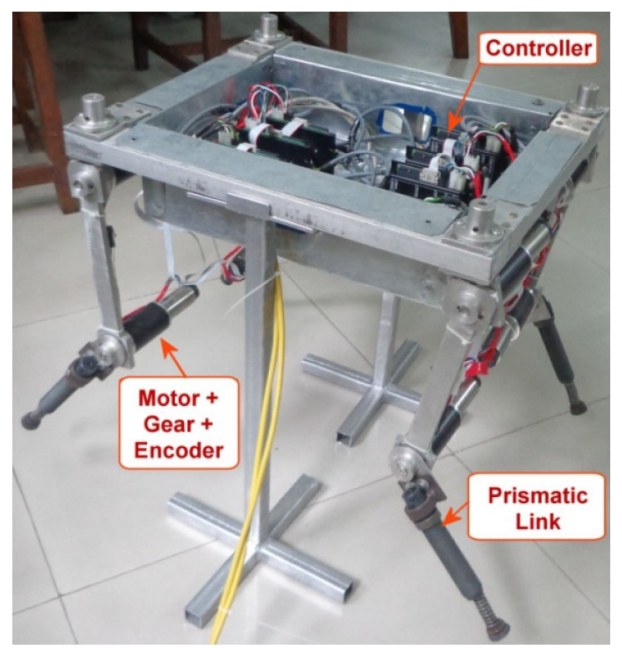

Figure 11. Quadruped robot on stand.

\subsection{Results}

The operation of the developed quadruped was tested for various gait patterns. Amble gait, which is statically stable gait in which legs operate one by one in 1-4-2-3 sequence, is considered in this paper as a test case. Here, experimental results are shown for amble gait. Figure 12 shows a few snaps of quadruped robot walking with amble gait. It takes $3.3 \mathrm{~s}$ to complete one cycle. Experiment data for joint rotations for five cycles are shown in figure 13.

Figure 12 shows robot forward motion. In five cycles, it is found to move by around $0.39 \mathrm{~m}$. Surface condition plays major role in the locomotion of robot. Also, there are always assumptions considered for preparation of mathematical model like mass center of link is located at the mid of its length, center of gravity of top body is located at the center of body, top body and upper links are rigid, joint rotation allows rotation of link about one axis only, robot is walking on hard surface and on even terrain and external force and moments effects are negligible. However, real situation differs.

\section{Locked joint fault accommodation}

\subsection{Strategies for locked joint fault tolerant}

A moving appendage device containing two-DOF planar robot with the payload at the end is introduced here to accommodate locked joint failure. Entire planar robot is arranged on the top of the quadruped robot body. The payload attached at the tip serves as an inertial element and its movement causes the change in the position of center of gravity (CG). Figure 14 shows the schematic diagram of a quadruped robot with moving appendage device in which $\{A\}$ is the inertial frame of reference and $\{B\}$ is the body frame. In addition, $\left\{P_{0}\right\}$ is the fixed frame which coincides with the body frame, $\left\{P_{1}\right\}$ is the frame at the joint between fixed frame and first link of two DOF robot, $\left\{P_{2}\right\}$ is the frame at the joint between first and second link, and $\left\{P_{3}\right\}$ is the frame attached at the tip of second link of two DOF planar robot.

Governing equations for angular velocity propagation (AVP) of planar robot can be derived as [26],

$$
{ }^{i+1}\left({ }^{A} \omega_{i+1}\right)={ }_{i}^{i+1} R^{i}\left({ }^{A} \omega_{i}\right)+{ }^{i+1}\left({ }^{i} \omega_{i+1}\right)
$$

where, ${ }^{i+1}\left({ }^{A} \omega_{i+1}\right)$ is the angular velocity of $(i+1)$ th link with respect to the inertial frame and expressed in $(i+1)$ th frame; ${ }_{i}^{i+1} R$ represents the transformation from the body frame $\{i\}$ to the frame $\{i+1\} ;{ }^{i}\left({ }^{A} \omega_{i}\right)$ is the angular velocity of the $i$ th link with respect to the inertial frame $\{A\}$ and expressed in $i$ th frame and ${ }^{i+1}\left({ }^{i} \omega_{i+1}\right)$ is the angular velocity of $(i+1)$ th link as observed from $i$ th link and expressed in $(i+1)$ th frame.

Equation (2) can be written for links 1 and 2 of two-DOF planar robot as,

$$
\begin{gathered}
{ }^{P_{1}}\left({ }^{A} \omega_{P_{1}}\right)={ }_{B}^{P_{1}} R^{B}\left({ }^{A} \omega_{B}\right)+{ }^{P_{1}}\left({ }^{B} \omega_{P 1}\right) \\
P_{2}\left({ }^{A} \omega_{P_{2}}\right)={ }_{P_{1}}^{P_{2}} R^{P_{1}}\left({ }^{A} \omega_{P_{1}}\right)+{ }^{P_{2}}\left({ }^{P_{1}} \omega_{P_{2}}\right)
\end{gathered}
$$

Frame $\left\{P_{3}\right\}$ has same angular velocity as frame $\left\{P_{2}\right\}$, because both these frames are attached on the same link. Thus,

$$
{ }^{P_{3}}\left({ }^{A} \omega_{P_{3}}\right)={ }^{P_{2}}\left({ }^{A} \omega_{P_{2}}\right)
$$

Here, $\quad P_{1}\left({ }^{B} \omega_{P_{1}}\right)=\left[\begin{array}{lll}0 & 0 & \dot{\theta}_{P_{1}}\end{array}\right]^{T} \quad$ and ${ }^{P_{2}}\left({ }^{P_{1}} \omega_{P_{2}}\right)=\left[\begin{array}{lll}0 & 0 & \dot{\theta}_{P_{2}}\end{array}\right]^{T}$, where $\dot{\theta}_{P_{1}}$ represents the angular velocity of the frame $\left\{P_{1}\right\}$ and similarly $\dot{\theta}_{P_{2}}$ represents angular velocity of the frame $\left\{P_{2}\right\}$. 

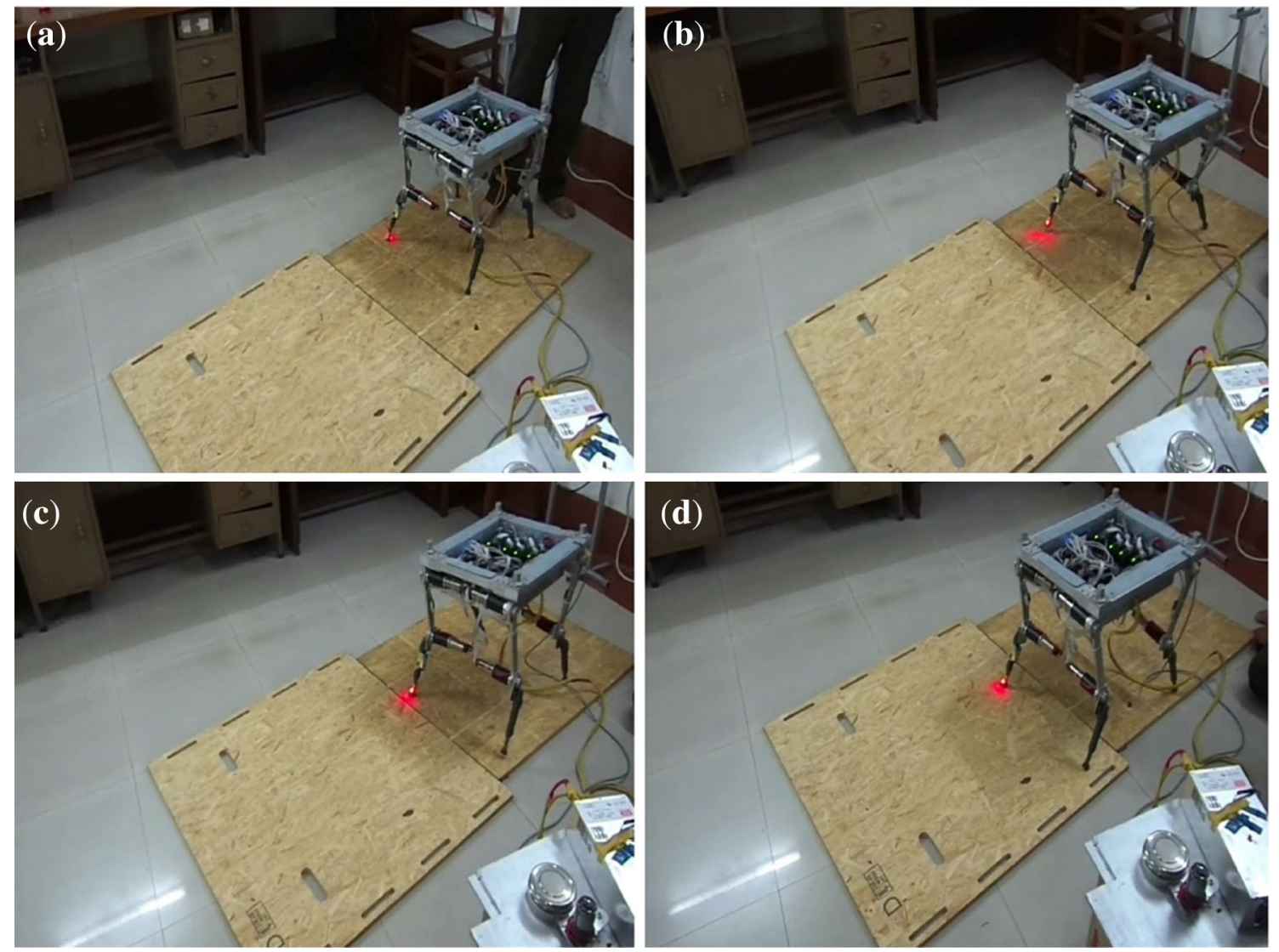

Figure 12. Quadruped robot walking.

For translational velocity propagation (TVP), the governing equations for the link tip velocity and the link CG velocity of planar robot can be given as [26],

$$
{ }^{A}\left({ }^{A} V_{i+1}\right)={ }^{A}\left({ }^{A} V_{i}\right)+{ }_{i}^{A} R\left[{ }^{i}\left({ }^{A} \omega_{i}\right) \times{ }^{i}\left({ }^{i} P_{i+1}\right)\right]
$$

where, ${ }^{A}\left({ }^{A} V_{i+1}\right)$ represent the translational velocity of frame $\{i+1\}$ with respect to inertial frame and expressed in the inertial frame; ${ }^{A}\left({ }^{A} V_{i}\right)$ represent the translational velocity of frame $\{i\}$ with respect to inertial frame and expressed in the inertial frame $;{ }^{i}\left({ }^{i} P_{i+1}\right)$ refers to the position vector of link frames $\{i+1\}$ with respect to its previous frames $\{i\}$ and expressed in frame $\{i\}$. Equation (6) can be written in for two-DOF planar robot as,

$$
\begin{gathered}
{ }^{A}\left({ }^{A} V_{P_{2}}\right)={ }^{A}\left({ }^{A} V_{P_{1}}\right)+{ }_{P_{1}}^{A} R\left[{ }^{P_{1}}\left({ }^{A} \omega_{P_{1}}\right) \times{ }^{P_{1}}\left({ }^{P_{1}} P_{P_{2}}\right)\right] \\
{ }^{A}\left({ }^{A} V_{P_{3}}\right)={ }^{A}\left({ }^{A} V_{P_{2}}\right)+{ }_{P_{2}}^{A} R\left[{ }^{P_{2}}\left({ }^{A} \omega_{P_{2}}\right) \times{ }^{P_{2}}\left({ }^{P_{2}} P_{P_{3}}\right)\right]
\end{gathered}
$$

If link lengths $l_{P 1}$ and $l_{P 2}$ are taken along the principal $X$ axis of the links then they can be represented in vector form as, $\quad{ }^{P_{1}} P_{P_{2}}=\left[\begin{array}{lll}l_{P 1} & 0 & 0\end{array}\right]^{T}, \quad{ }^{P_{2}} P_{P_{3}}=\left[\begin{array}{lll}l_{P 2} & 0 & 0\end{array}\right]^{T}$. The moving appendage device is activated only when fault occurs. If one of the legs joint is locked then the leg can still move in a restricted way with the help of the other working joint in that leg. Assuming that robot is walking with trot gait. In the first phase of trot gait, diagonally opposite legs move forward while in the second phase, the same leg tips touch the ground and body propagation takes place. In the case of joint failure, there is no guarantee of proper leg tip contact on the ground for body propagation and thus the locomotion is severely affected. In the proposed control strategy, the two-DOF robot payload position is controlled in such a way that it moves towards the hip joint of failed leg during body propagation to ensure proper contact of failed leg tip on the ground and towards center of the body during the forward movement phase of the failed leg.

For example, if joint 1 of leg 1 has failed then the desired $X$ and $Y$ coordinates of payload mass during the first phase, i.e., during leg 1 forward movement, are $X_{\text {des }}=-0.098 \mathrm{~m}$ and $Y_{d e s}=-0.013 \mathrm{~m}$ in $X$ and $Y$ directions, respectively. These limits are decided from constrain of physical model. The main purpose is to keep the mass near the center during first phase while during the second phase, i.e., contact of leg tip with the ground,

$$
X_{d e s}=\left(l_{P 1}+l_{P 2}-0.005\right) \sin \left(\tan ^{-1}\left(\frac{R_{1 x}}{R_{1 y}}\right)\right)
$$



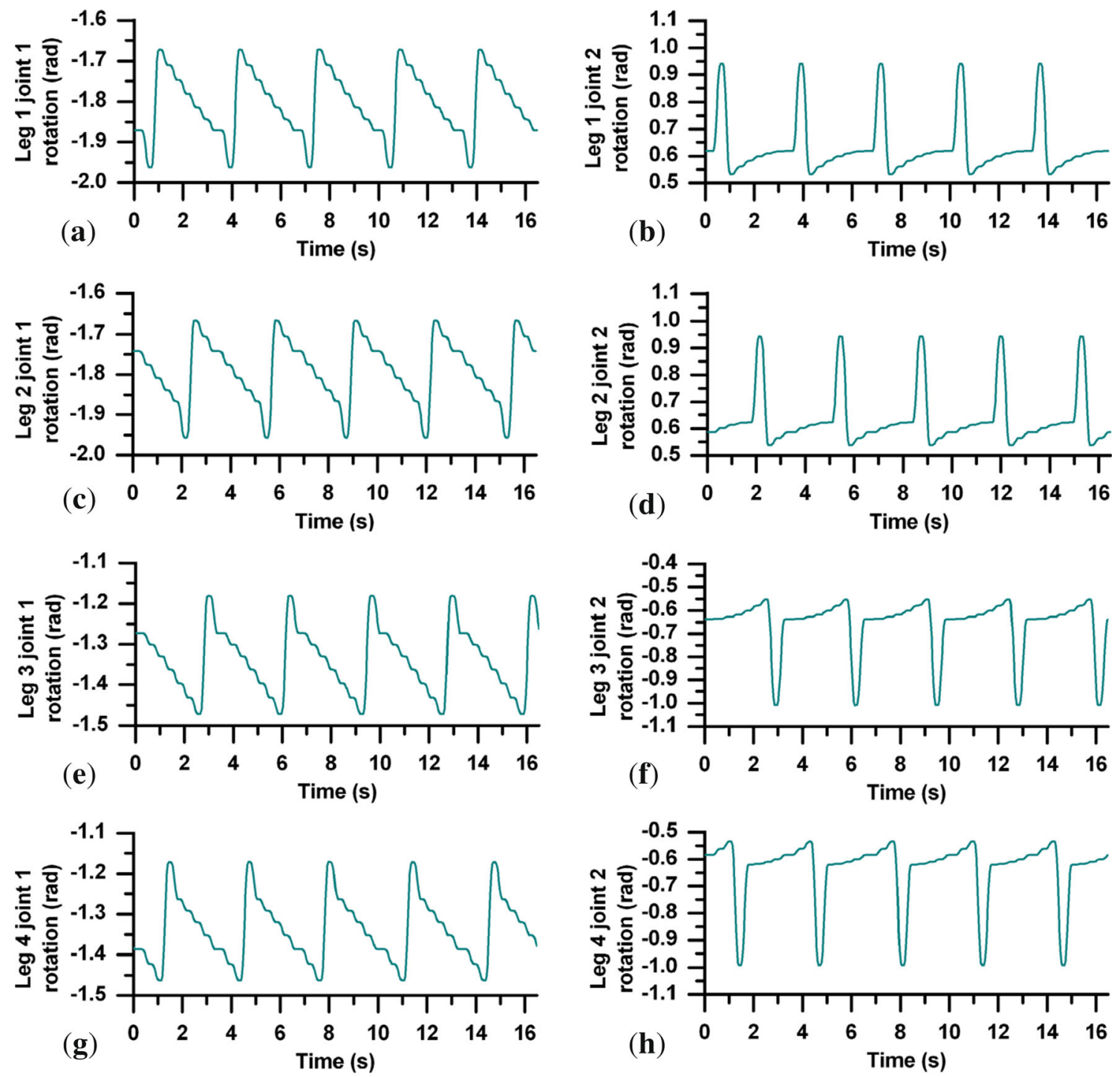

Figure 13. Joints rotation: (a) Leg 1 joint 1; (b) Leg 1 joint 2; (c) Leg 2 joint 1; (d) Leg 2 joint 2; (e) Leg 3 joint 1 ; (f) Leg 3 joint 2 ; (g) Leg 4 joint 1; (h) Leg 4 joint 2.

$$
Y_{d e s}=\left(l_{P 1}+l_{P 2}-0.005\right) \cos \left(\tan ^{-1}\left(\frac{R_{1 x}}{R_{1 y}}\right)\right)
$$

where, $R_{1 x}$ and $R_{1 y}$ are the distance of hip joint from body center. To avoid singularity problems, an offset of $0.005 \mathrm{~m}$ is introduced in Eqs. (9) and (10). Similar control laws are expressed for joint failure in other legs. The above discussed control law is activated only after a joint failure is detected in a particular leg. There is a bank of control laws implemented in the form of if-then blocks and the appropriate control law is executed by the decision support system based on the results of the fault diagnosis tools in the system supervision module.

For the above desired coordinates of a planar robot tip, necessary joint rotations can be derived using the Jacobian. The Jacobian is the mapping between velocities in the joint space to the Cartesian space. Equation (8) is utilized to

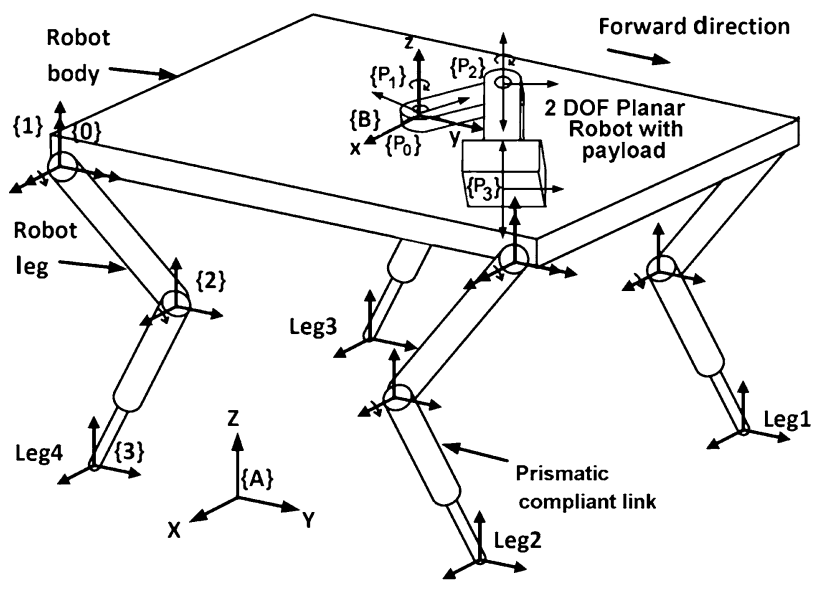

Figure 14. Schematic diagram of a quadruped robot with moving appendage device. 


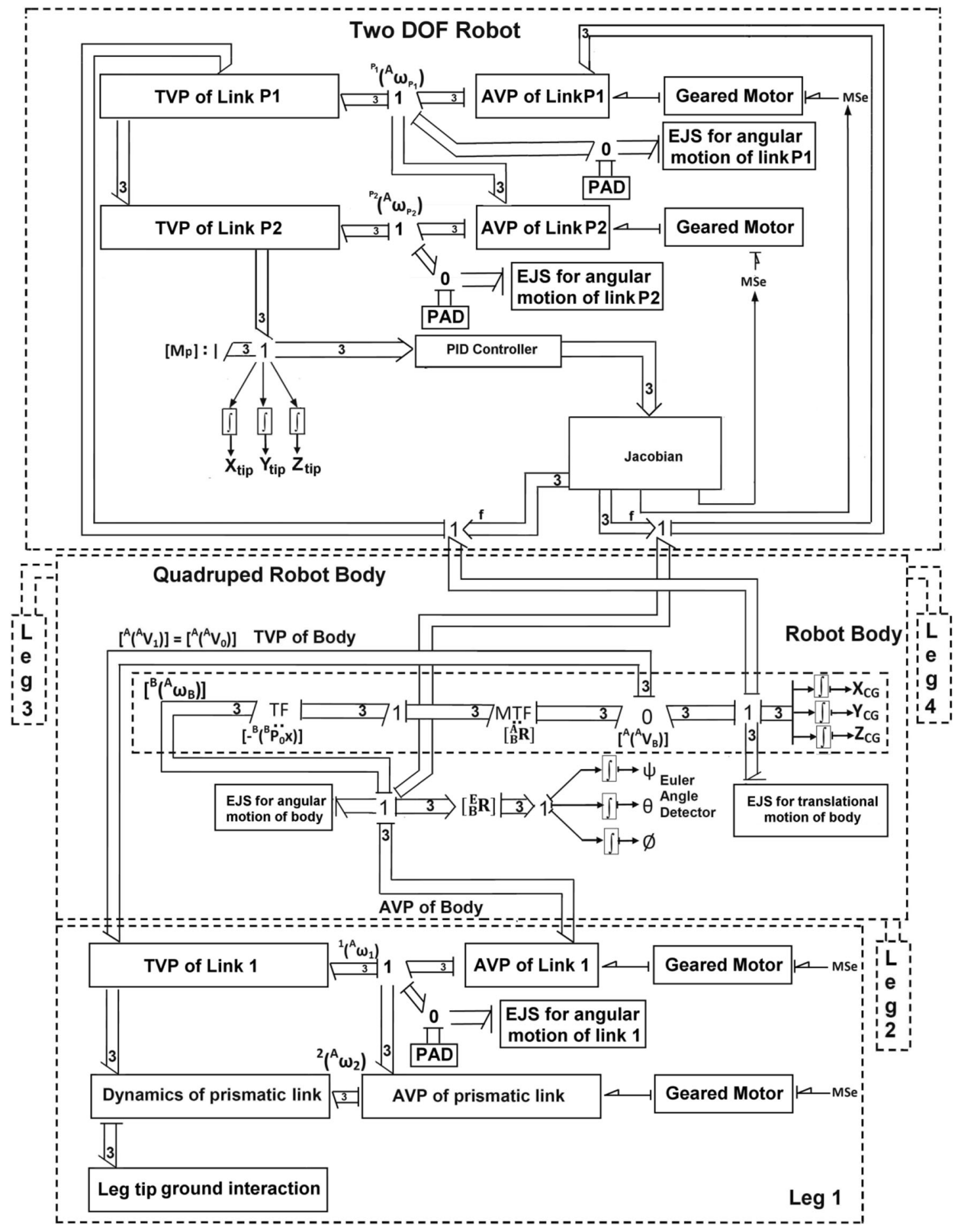

Figure 15. Multi-bond graph of quadruped robot with two-DOF planar robot.

derive the Jacobian matrix. For position control of twoDOF planar robot tip with reference to time, desired position of the tip discussed above are used as the reference. Here, the reference velocity is compared with the actual planar robot tip velocity and error values are sent to a PID controller. The PID controller sends corrective signals to the Jacobian which then evaluates the required efforts at the joints for appendage movement. The required efforts for the first and second joints can be evaluated as

$$
V_{1}=C_{X} \beta_{12}+C_{Y} \beta_{11}+C_{Z} \beta_{10}
$$


Table 4. Input parameters.

\begin{tabular}{|c|c|}
\hline Parameters & Value \\
\hline \multicolumn{2}{|l|}{ Leg parameters } \\
\hline First link length of leg $\left(l_{1}\right)$ & $0.225 \mathrm{~m}$ \\
\hline Mass of first link $\left(M_{l 1}\right)$ & $1.11 \mathrm{~kg}$ \\
\hline Mass of cylinder part of the prismatic link $\left(M_{c}\right)$ & $0.3 \mathrm{~kg}$ \\
\hline Mass of piston part of the prismatic link $\left(M_{p}\right)$ & $0.2 \mathrm{~kg}$ \\
\hline \multicolumn{2}{|l|}{ Inertia of Link 1} \\
\hline$I_{x x 1}$ & $0.013346 \mathrm{~kg} \mathrm{~m}^{2}$ \\
\hline$I_{y y 1}$ & $0.007396 \mathrm{~kg} \mathrm{~m}^{2}$ \\
\hline$I_{z z 1}$ & $0.011563 \mathrm{~kg} \mathrm{~m}^{2}$ \\
\hline \multicolumn{2}{|l|}{ Inertia of cylinder part of the prismatic link } \\
\hline$I_{x x c}=I_{z z c}$ & $0.005144 \mathrm{~kg} \mathrm{~m}^{2}$ \\
\hline$I_{y y c}$ & $0.000487 \mathrm{~kg}^{2}$ \\
\hline \multicolumn{2}{|l|}{ Inertia of piston and piston rod of the prismatic link } \\
\hline$I_{x x p}=I_{z z p}$ & $0.00168 \mathrm{~kg} \mathrm{~m}^{2}$ \\
\hline$I_{y y p}$ & $0.000025 \mathrm{~kg} \mathrm{~m}^{2}$ \\
\hline Stiffness of the prismatic link $\left(k_{f}\right)$ & $5000 \mathrm{~N} / \mathrm{m}$ \\
\hline Damping of the prismatic link $\left(R_{f}\right)$ & $274 \mathrm{Ns} / \mathrm{m}$ \\
\hline Contact point stiffness at the piston cylinder of the prismatic link $\left(k_{b}\right)$ & $10^{8} \mathrm{~N} / \mathrm{m}$ \\
\hline Contact point resistance at the piston and cylinder of the prismatic link $\left(R_{b}\right)$ & $10^{3} \mathrm{Ns} / \mathrm{m}$ \\
\hline Length of piston and piston rod of the prismatic link $\left(l_{p}\right)$ & $0.1 \mathrm{~m}$ \\
\hline Distance of cylinder CG from the end frame of prismatic link $\left(l_{c g}\right)$ & $0.05 \mathrm{~m}$ \\
\hline Distance of piston CG from the end frame of the prismatic link $\left(l_{p g}\right)$ & $0.07 \mathrm{~m}$ \\
\hline Mass of piston \& piston rod of the prismatic link $\left(m_{p}\right)$ & $0.2 \mathrm{~kg}$ \\
\hline Mass of cylinder part of the prismatic link $\left(m_{c}\right)$ & $0.3 \mathrm{~kg}$ \\
\hline Position of the cylinder end point with respect to the body fixed frame at the mass center in meter, $\left(x_{2}, y_{2}, z_{2}\right)$ & $(0.0,-0.05,0.0)$ \\
\hline Position of the piston end point with respect to the body fixed frame at the mass center in meter, $\left(x_{3}, y_{3}, z_{3}\right)$ & $(0.0,0.07,0.0)$ \\
\hline \multicolumn{2}{|l|}{ Common parameters } \\
\hline Mass of body $(M b)$ & $6.94 \mathrm{~kg}$ \\
\hline \multicolumn{2}{|l|}{ Inertia of body } \\
\hline$I_{x b}$ & $0.1470 \mathrm{~kg} \mathrm{~m}^{2}$ \\
\hline$I_{y b}$ & $0.1045 \mathrm{~kg} \mathrm{~m}^{2}$ \\
\hline$I_{z b}$ & $0.2466 \mathrm{~kg} \mathrm{~m}^{2}$ \\
\hline Ground damping in $x, y, z$ direction $\left(R_{g x}, R_{g y}, R_{g z}\right)$ & $1000 \mathrm{Ns} / \mathrm{m}$ \\
\hline Ground stiffness in $z$ direction $\left(K_{g z}\right)$ & $10^{6} \mathrm{~N} / \mathrm{m}$ \\
\hline \multicolumn{2}{|l|}{ Controller parameters } \\
\hline Proportional gain of controller $\left(K_{p}\right)$ & 150 \\
\hline Derivative gain of controller $\left(K_{d}\right)$ & 90 \\
\hline Integral gain of controller $\left(K_{i}\right)$ & 40 \\
\hline \multicolumn{2}{|l|}{ Joint actuator parameters of quadruped } \\
\hline Motor constant $\left(K_{t}\right)$ & $0.0276 \mathrm{Nm} / \mathrm{A}$ \\
\hline Motor armature resistance $\left(R_{m}\right)$ & $0.386 \Omega$ \\
\hline Motor inductance $\left(I_{m}\right)$ & $0.001 \mathrm{H}$ \\
\hline Gear ratio $(n)$ & 230 \\
\hline \multicolumn{2}{|l|}{ Two-DOF planar robot parameters } \\
\hline Link 1 and Link 2 length of planar robot $\left(l_{P 1}, l_{P 2}\right)$ & $0.055 \mathrm{~m}, 0.140 \mathrm{~m}$ \\
\hline Mass of link of planar robot $\left(M_{P l 1}, M_{P l 2}\right)$ & $0.05 \mathrm{~kg}, 0.06 \mathrm{~kg}$ \\
\hline Payload mass $\left(M_{p}\right)$ & $0.5 \mathrm{~kg}$ \\
\hline Inertia of both link of planar robot $\left(I_{x}, I_{y}, I_{z}\right)$ & $0.0001 \mathrm{~kg} \mathrm{~m}^{2}$ \\
\hline Motor constant $\left(K_{t}\right)$ & $0.02 \mathrm{Nm} / \mathrm{A}$ \\
\hline Motor armature resistance $\left(R_{a}\right)$ & $0.1 \mathrm{Ohms}$ \\
\hline Gear ratio & 100 \\
\hline Bearing resistance $\left(R_{b}\right)$ & $0.01 \mathrm{Ns} / \mathrm{m}$ \\
\hline Proportional and integral gain values $\left(K_{p p}\right),\left(K_{i p}\right)$ & 250,6700 \\
\hline
\end{tabular}



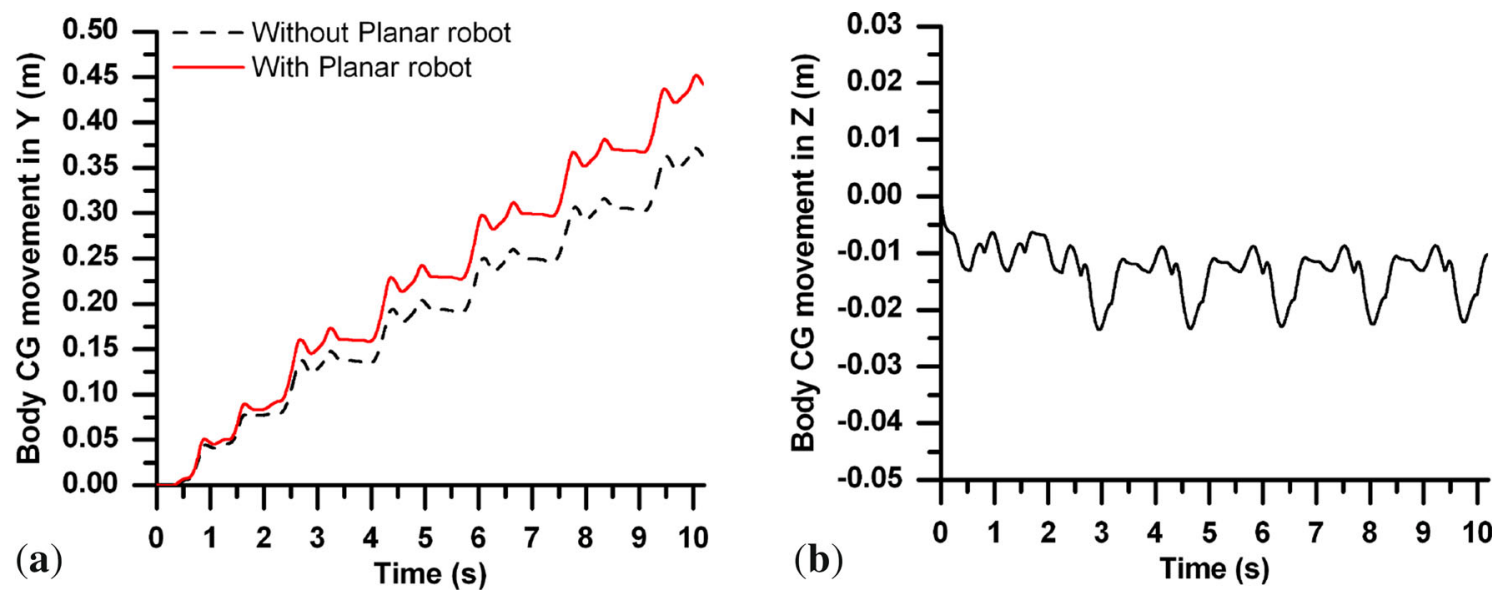

Figure 16. Body $\mathrm{CG}$ movement in (a) $\mathrm{Y}$ and (b) $\mathrm{Z}$ directions.
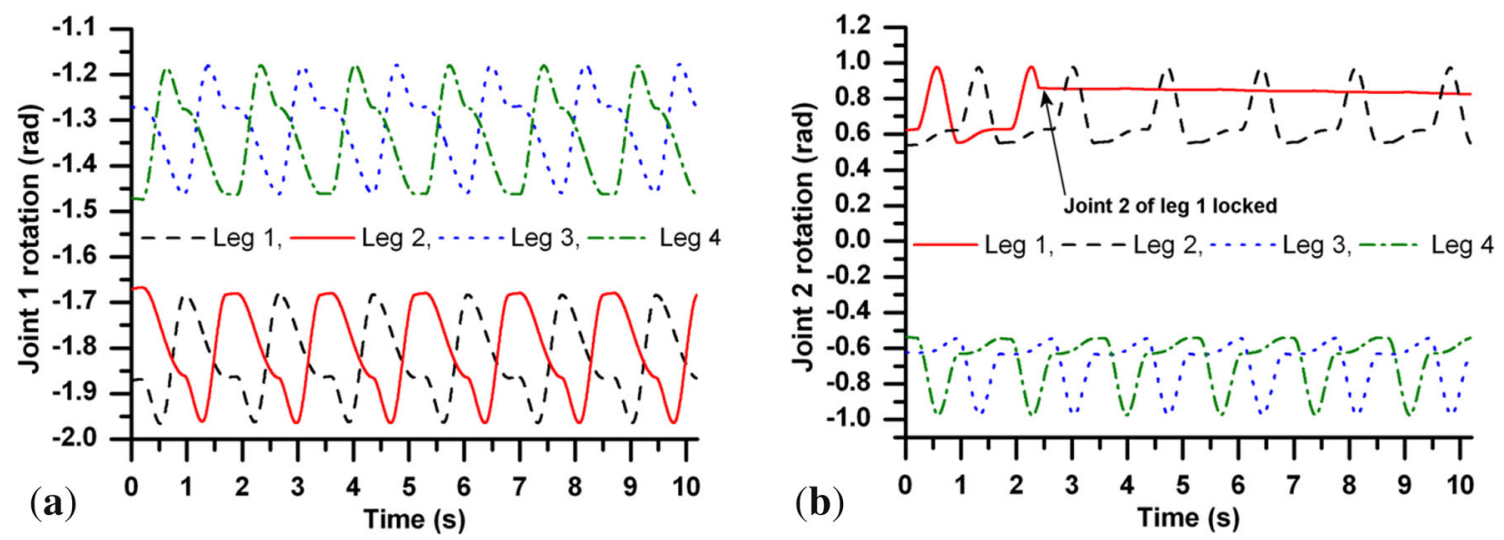

Figure 17. Joints rotation: (a) Joint 1; (b) Joint 2.
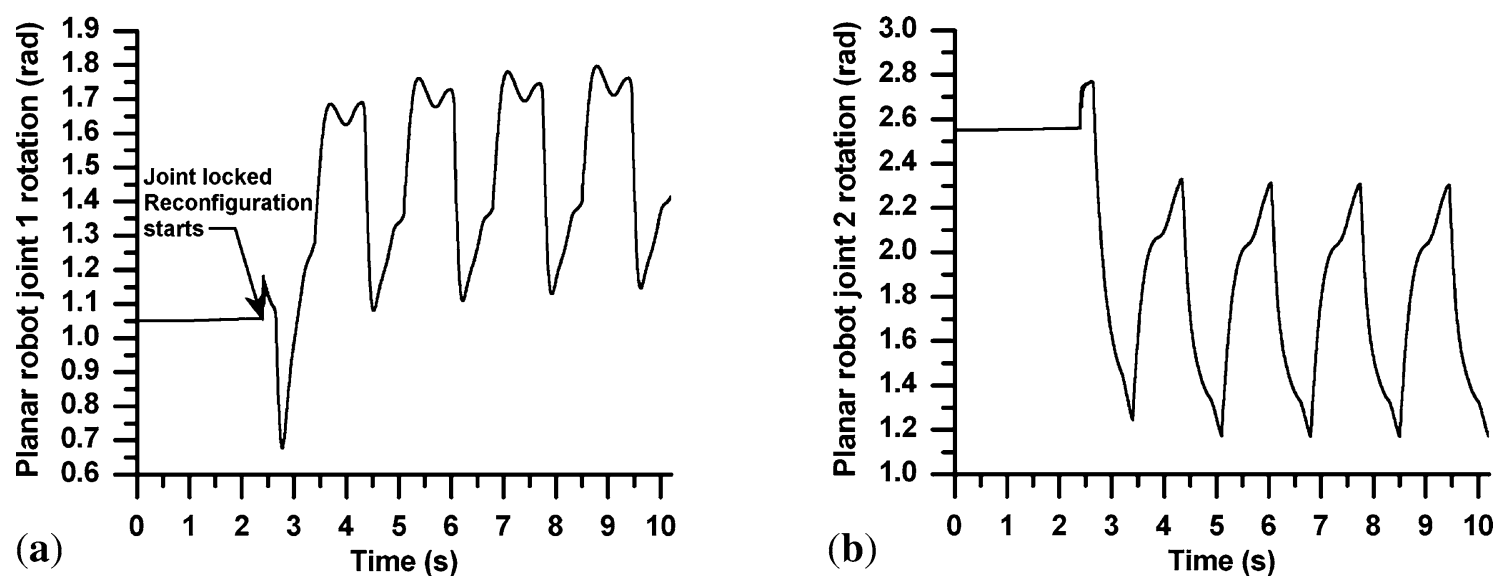

Figure 18. Two-DOF Planar robot joints rotation: (a) Joint 1; (b) Joint 2. 

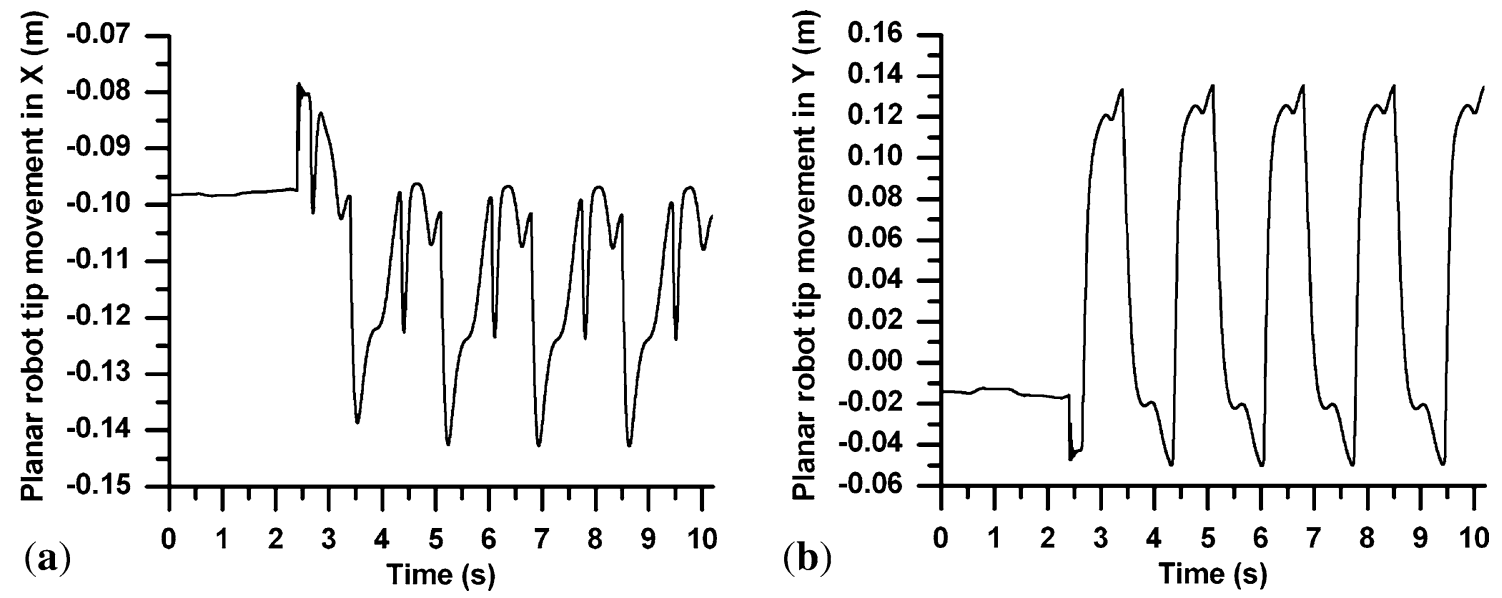

Figure 19. Two-DOF Planar robot tip movement in (a) $\mathrm{X}$ and (b) $\mathrm{Y}$ direction.

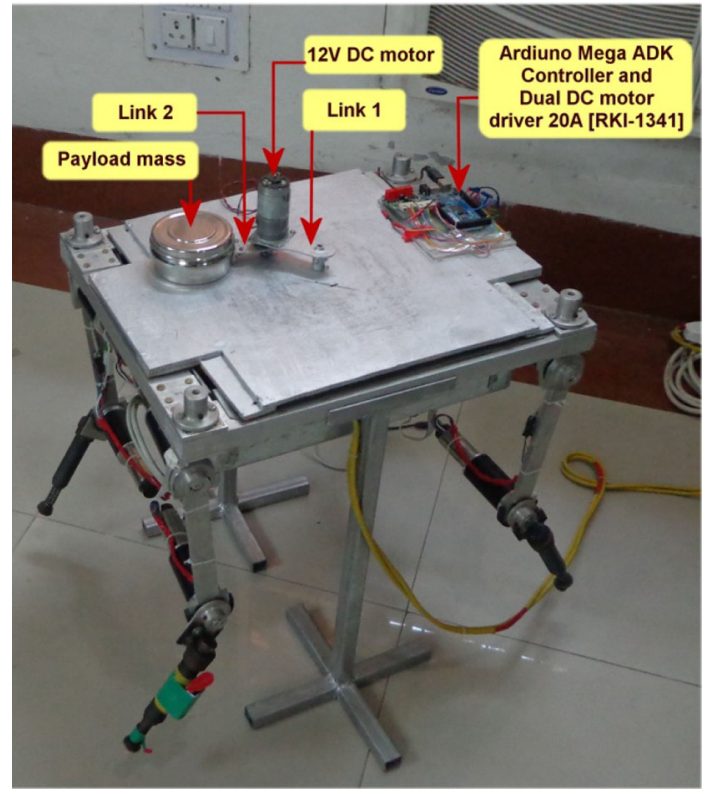

Figure 20. Experiment set-up.

$$
V_{2}=C_{X} \beta_{15}+C_{Y} \beta_{14}+C_{Z} \beta_{13}
$$

Where $C_{X}, C_{Y}$ and $C_{Z}$ are corrective signal received from PID in $X, Y$ and $Z$ directions, respectively. Detailed description of coefficients $\beta_{10}, \beta_{11}, \beta_{12}, \beta_{13}, \beta_{14}$ and $\beta_{15}$ used in Eqs. (11) and (12) are shown in "Appendix B".

\subsection{Dynamic model of the robot}

To validate the above discussed reconfiguration strategy, a bond graph model is developed. Bond graph is a tool used to model physical systems through power interaction. Multi-bond graph presentation of quadruped robot with two-DOF planar robot is shown in figure 15. A compact and a simple presentation of a bond graph model can be carried out in multi bond graph form. Modeling of a quadruped robot consists of a modeling of translational and angular dynamics of robot body and legs. The detailed discussion of bond graph model of quadruped robot is covered in our previous publications $[13,14]$. Model of the two-DOF planar robot, discussed in Sect. 3.1, is appended on the bond graph model of the quadruped robot given in $[13,14]$. Using common flow junction (1 junction), common effort junction (0 junction), one port elements viz., Resistor (R), Inertia (I), Capacitor (C), Source of Effort (SE) and Source of Flow (SF); two port elements viz., transformer (TF) and gyrator (GR), the bond graph model of the two DOF planar robot is developed. As shown in figure 15, AVP of link P1 indicates modeling of angular velocity propagation of P1 link as per Eq. (3), and AVP of link P2 indicates modeling of angular velocity propagation of P2 link Eq. (4). Similarly, TVP of links P1 and P2 indicate modeling of translational velocity propagations as per Eqs. (7) and (8), respectively, for links P1 and P2. Modeling of geared motors of two-DOF robot is same as modeling of geared motor of quadruped robot discussed in our publication [14]. The translational velocity and the angular velocity vectors have been resolved into three mutually perpendicular components, and their dynamics can be modelled with Euler junction structure (EJS). EJS of two DOF planar link P1 and P2 is modelled as same as EJS of quadruped robot link discussed in [14]. The pad is used to avoid differential causality. Pads are artificial compliances/lumped flexibilities that can be used in bond graph. The Jacobian sub-model shows the mapping between velocities in the joint space to the Cartesian space. This sub model can be developed using Eq. (8). PID controller sub model as shown in figure 15 is also developed which is responsible for the required effort at joint P1 and P2 of two DOF planar robot. PID controller sub model is developed using Eqs. (11) and (12). 

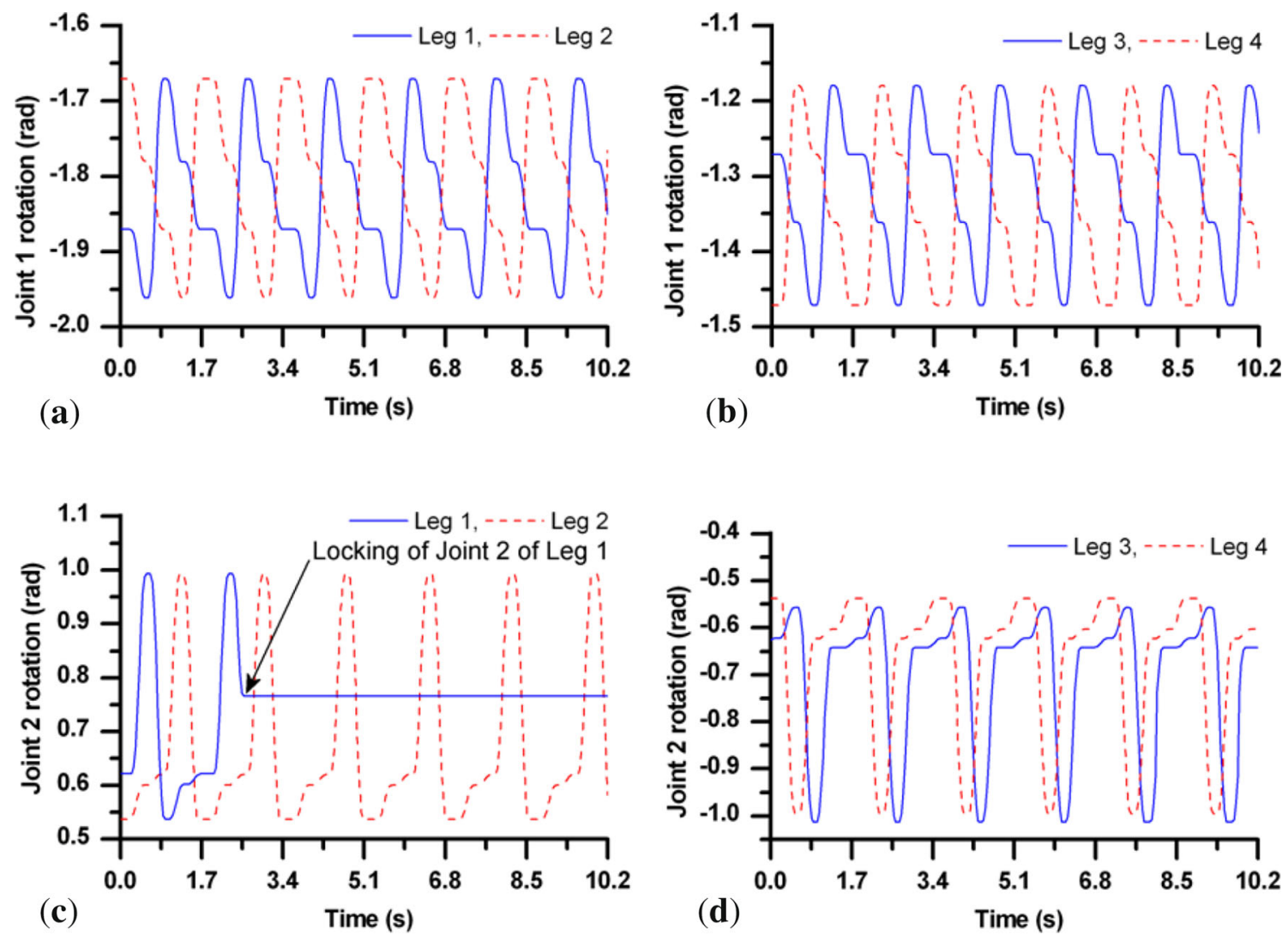

Figure 21. Joints rotation of physical model of quadruped robot during experiment: (a) joint 1 of leg 1 and 2; (b) joint 1 of leg 3 and 4; (c) joint 2 of leg 1 and 2; (d) joint 2 of leg 3 and 4 .
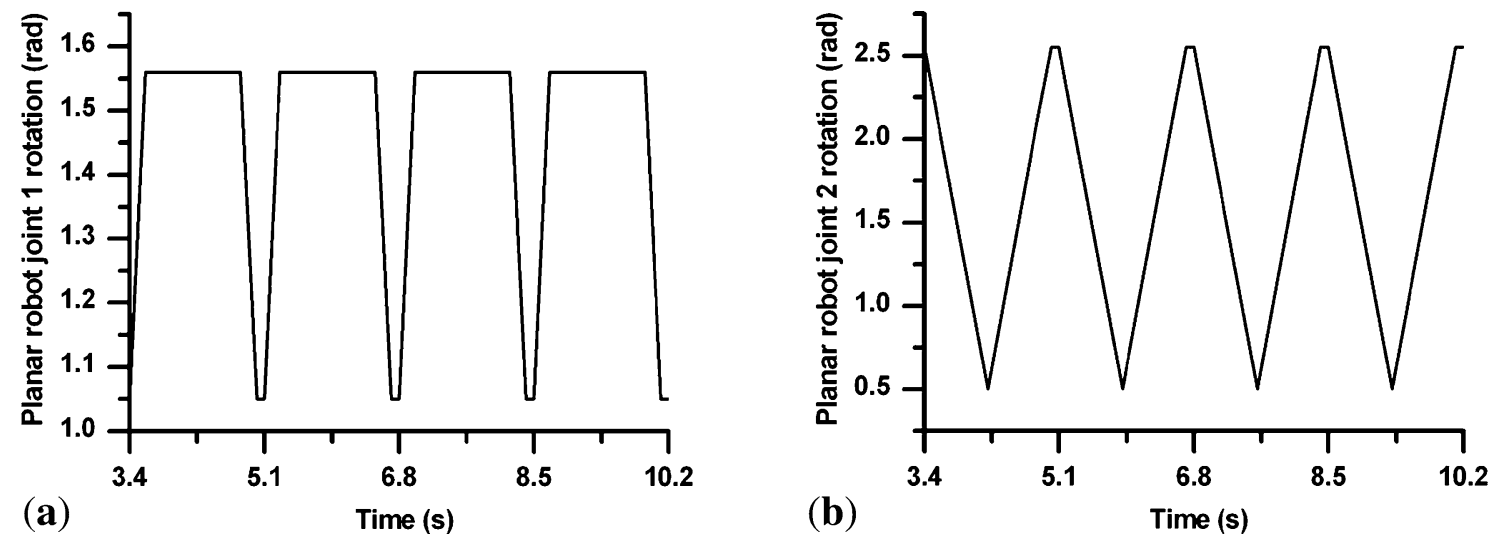

Figure 22. Joint rotation of physical model of two-DOF planar robot during experiment: (a) joint 1; (b) joint 2.

\subsection{Simulation results of locked joint fault accommodation through reconfiguration}

The control law discussed in section 3.1 for fault accommodation through reconfiguration is implemented in the model developed in section 3.2. Parameter used for the simulations are listed in table 4.

A fault is intentionally introduced during the locomotion of the quadruped robot and the locomotion performance is observed with and without the moving appendage.
Simulation is carried out for six cycles. Each cycle is of $1.7 \mathrm{~s}$ duration. Initially, it is assumed that there is no fault. Figure 16 shows the quadruped body $C G$ motion in the $Y$ and $Z$ directions. Figures 17(a) and (b) show the joint 1 and 2 rotations, respectively, of all leg joints of the quadruped. Initially, all the joints are working properly and the moving appendage device is switched off. Immediately after the joint failure (joint locked), the moving appendage device starts working. For demonstration of the control 

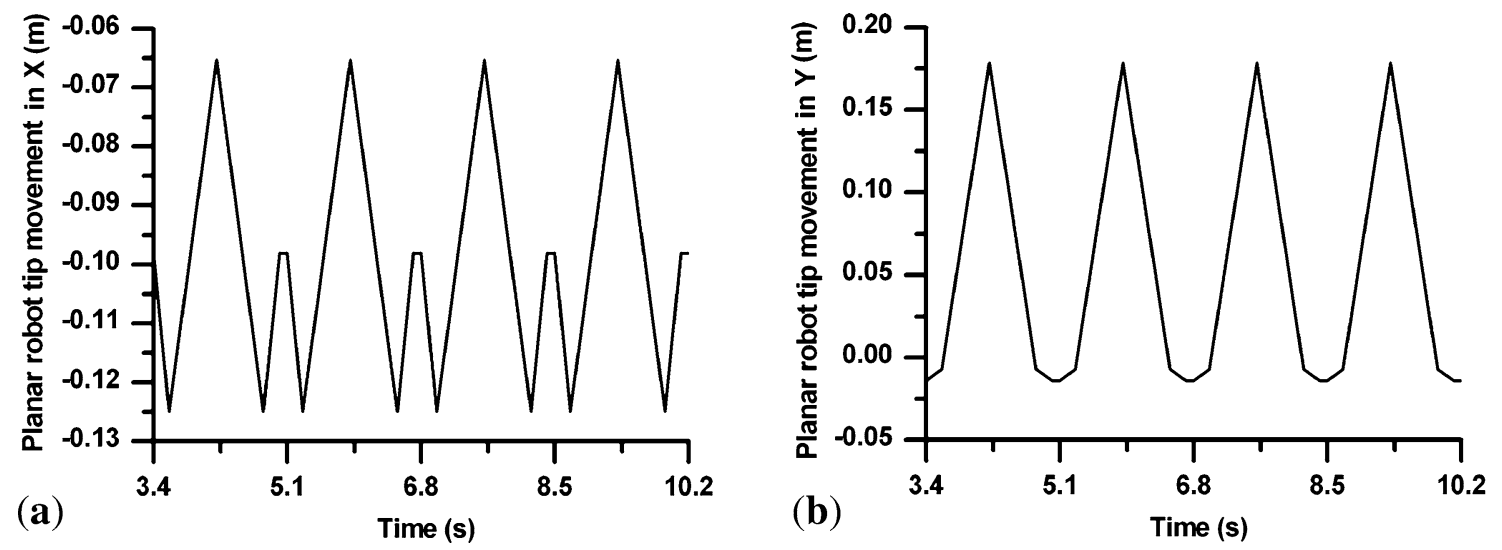

Figure 23. Tip movement of physical model of two-DOF planar robot during experiment in (a) X and (b) Y.
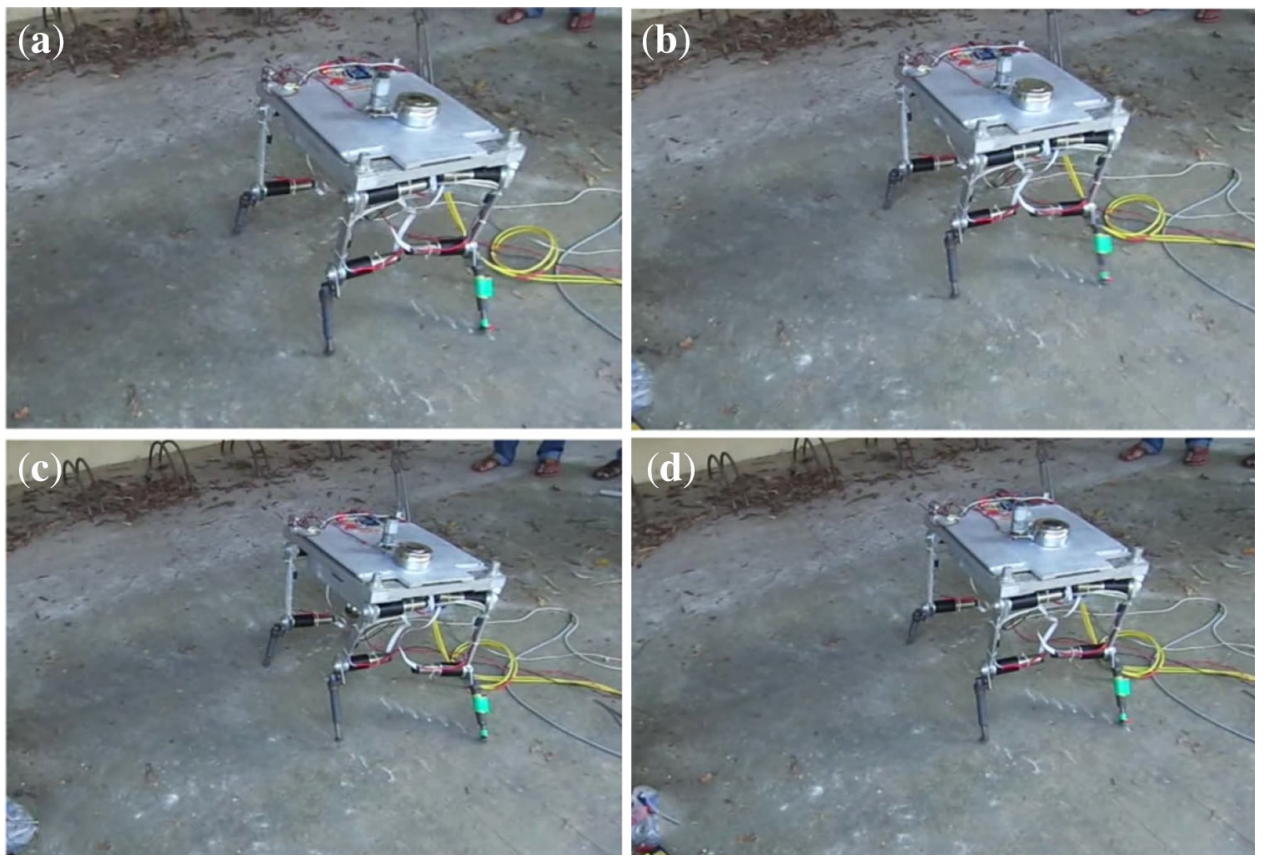

Figure 24. Snaps taken during experiment of reconfiguration using two-DOF planar robot.

strategy, the locked joint failure is introduced at joint 2 of leg 1 . Figure 18 shows two-DOF planar robot joint rotation for joint 1 and 2, while figure 19 shows the planar robot tip position in the $X$ and $Y$ local frames with respect to time, respectively, in which the moving appendage starts working immediately after the failure is detected at $2.4 \mathrm{~s}$ (automatic failure detection is not implemented here, so the switching condition is hard-coded). As per the control strategy, the appendage device remains near the center during leg 1 forward motion, while it tries to move towards the hip joint of leg 1 during body propagation. Because of the weight of the payload mass, the controller ensures proper contact of leg tip with the ground and effective body propagation. If the quadruped robot continues locomotion without contribution of the moving appendage device then it can traverse $0.362 \mathrm{~m}$ distance, whereas it can travel up to $0.441 \mathrm{~m}$ in $10.2 \mathrm{~s}$ with the controlled motion of the moving appendage. Thus, it shows overall improvement in locomotion because of the control strategies implemented with the moving appendage device.

\subsection{Experimental validation of locked joint fault accommodation through reconfiguration}

Above discussed simulation results are also verified through experimental results. A Quadruped robot as discussed in section 2 is used. A two-DOF planar robot with a 
payload at its end is constructed and mounted on the quadruped robot as shown in figure 20. Links of this planar robot are fabricated from mild-steel and $0.5 \mathrm{~kg}$ mass is kept in stainless box attached at the end of robot. Both the links are operated through $12 \mathrm{~V}$ DC motor (Brushed DC motor with 100:1 metal gearbox) attached at their joints. These motors get power through 20 A Dual DC motor driver which is controlled by Ardiuno Mega ADK controller.

Leg joints rotations are shown in figure 21 for both healthy and faulty state while planar robot joint rotations are shown in figure 22 for faulty state and robot tip position in $X$ and $Y$ directions are shown in figures 23(a) and (b), respectively. It has been observed that without such moving appendage device with failed leg quadruped travels around $0.3 \mathrm{~m}$ distance in five cycles while with appendage device it travels around $0.35 \mathrm{~m}$ distance. Figure 24 shows few snaps taken during experiment on reconfiguration for locked joint failure using two DOF planar robot. Figure 24(a) shows planar robot payload position near the center, Figures 24(b) and (c) payload moves away from the center and figure 24(d) shows payload moves near the center.

\section{Conclusions}

This paper presents the entire process of development of a quadruped robot. This work may serve as a guideline for those who are interested to develop quadruped robot for their research purpose. Here, experimental results of robot walking with amble gait are demonstrated. There are certain issues like body disturbance during dynamic walk and different types of joint failures. A strategy for locked joint failure through reconfiguration by using hardware redundancy is demonstrated. Simulation and experimental results show the success of developed fault tolerant control strategy. This quadruped robot with the added two-DOF robot on its platform or elsewhere may be used to explore various other control strategies such as posture control, fault tolerant and reconfiguration strategies for other types of joint failures and/or multiple joint failures.

\section{Acknowledgements}

The research work presented here was funded by the Department of Science and Technology (DST), India under Indo-Korea Joint Research in Science and Technology vide Grant No. INT/Korea/P-13. Mr M M Gor is thankful to G. H. Patel College of Engineering and Technology, Gujarat, India for allowing him to carry out research work at IIT Roorkee. The work of Mr J M Yang and Mr S W Kwak was supported by the Korea government (MEST) vide the National Research Foundation of Korea Grant No. NRF2011-0027705.

\section{Appendix A}

Equation of motions for back stance phase and front stance phase

$$
\begin{aligned}
& \tau_{1}=\left[-l_{1}\left(m_{b}+m_{1}+m_{2}\right) \sin \left(\theta_{2}\right)\right] \ddot{l}_{2}+\left[-l_{1}^{2}\left(m_{b}+m_{1}+m_{2}\right)\right. \\
& -l_{2}^{2}\left(m_{b}+2 m_{1}+m_{2}\right)-L l_{1}\left(m_{b}+2 m_{1}+2 m_{2}\right) \sin \left(\theta_{1}\right) \\
& -2 l_{1} l_{2}\left(m_{b}+m_{1}+m_{2}\right) \cos \left(\theta_{2}\right) \\
& +\left(m_{1}+m_{2}\right) l_{1}^{2} \cos \left(\theta_{1}+\theta_{3}\right) \\
& +\left(m_{1}+m_{2}\right) l_{1} l_{2} \cos \left(\theta_{1}-\theta_{2}+\theta_{3}\right) \\
& +m_{2} l_{1} l_{0} \cos \left(\theta_{1}+\theta_{3}-\theta_{4}\right) \\
& +m_{2} l_{2} l_{0} \cos \left(\theta_{2}-\theta_{1}+\theta_{4}-\theta_{3}\right) \\
& -L l_{2}\left(m_{b}+2 m_{1}+2 m_{2}\right) \\
& \left.\sin \left(\theta_{1}-\theta_{2}\right)\right] \ddot{\theta}+\left[l_{1}^{2}\left(m_{b}+m_{1}+m_{2}\right)+m_{1} r_{1}^{2}\right. \\
& +l_{2}^{2}\left(m_{b}+2 m_{1}+m_{2}\right)+2 l_{1} l_{2}\left(m_{b}+m_{1}+m_{2}\right) \\
& \left.\cos \left(\theta_{2}\right)\right] \ddot{\theta}_{1}+\left[-l_{2}^{2}\left(m_{b}+2 m_{1}+m_{2}\right)\right. \\
& \left.-l_{1} l_{2}\left(m_{b}+m_{1}+m_{2}\right) \cos \left(\theta_{2}\right)\right] \ddot{\theta}_{2} \\
& -2\left(m_{b}+2 m_{1}+m_{2}\right) l_{2} \dot{l}_{2}\left(\dot{\theta}+\dot{\theta}_{2}-\dot{\theta}_{1}\right) \\
& +\left(m_{b}+2 m_{1}+2 m_{2}\right) \\
& {\left[-L l_{1} \dot{\theta}^{2} \cos \left(\theta_{1}\right)-L l_{2} \dot{\theta}^{2} \cos \left(\theta_{1}-\theta_{2}\right)\right]} \\
& +\left(m_{b}+m_{1}+m_{2}\right) l_{1}\left[\dot{l}_{2}\left(2 \dot{\theta}_{2}-2 \dot{\theta}_{1}+2 \dot{\theta}\right) \cos \left(\theta_{2}\right)\right. \\
& \left.+l_{2} \dot{\theta}_{2}\left(\dot{\theta}_{2}-2 \dot{\theta}_{1}+2 \dot{\theta}\right) \sin \left(\theta_{2}\right)\right] \\
& +\left(m_{1}+m_{2}\right)\left[-l_{1}^{2} \dot{\theta}^{2} \sin \left(\theta_{1}+\theta_{3}\right)\right. \\
& \left.-l_{1} l_{2} \dot{\theta}^{2} \sin \left(\theta_{1}-\theta_{2}+\theta_{3}\right)\right] \\
& +m_{2}\left[-l_{1} l_{0} \dot{\theta}^{2} \sin \left(\theta_{1}+\theta_{3}-\theta_{4}\right)\right. \\
& \left.+l_{0} l_{2} \dot{\theta}^{2} \sin \left(\theta_{2}-\theta_{1}+\theta_{4}-\theta_{3}\right)\right] \\
& +g\left[-\left(m_{b}+m_{1}+m_{2}\right) l_{1} \sin \left(\theta_{1}-\left(\theta+\theta_{p}\right)\right)\right. \\
& \left.+\left(m_{b}+2 m_{1}+m_{2}\right) l_{2} \sin \left(\theta_{2}-\theta_{1}+\theta+\theta_{p}\right)\right] \\
& \tau_{2}=\left[\left(m_{b}+2 m_{1}+m_{2}\right) l_{2}^{2}+\left(m_{b}+2 m_{1}+2 m_{2}\right) L l_{2}\right. \\
& \sin \left(\theta_{1}-\theta_{2}\right)+\left(m_{b}+m_{1}+m_{2}\right) l_{1} l_{2} \cos \left(\theta_{2}\right) \\
& -\left(m_{1}+m_{2}\right) l_{1} l_{2} \cos \left(\theta_{1}-\theta_{2}+\theta_{3}\right) \\
& \left.-m_{2} l_{0} l_{2} \cos \left(\theta_{2}-\theta_{1}+\theta_{4}-\theta_{3}\right)\right] \ddot{\theta} \\
& +\left[-l_{2}^{2}\left(m_{b}+2 m_{1}+m_{2}\right)-l_{1} l_{2}\left(m_{b}+m_{1}+m_{2}\right)\right. \\
& \left.\cos \left(\theta_{2}\right)\right] \ddot{\theta}_{1}+\left[l_{2}^{2}\left(m_{b}+2 m_{1}+m_{2}\right)+m_{2} r_{2}^{2}\right] \ddot{\theta}_{2} \\
& +\left(m_{b}+2 m_{1}+2 m_{2}\right) L l_{2} \dot{\theta}^{2} \cos \left(\theta_{1}-\theta_{2}\right) \\
& +\left(m_{b}+m_{1}+m_{2}\right) l_{1} l_{2}\left(\dot{\theta}-\dot{\theta}_{1}\right)^{2} \sin \left(\theta_{2}\right) \\
& +\left(m_{1}+m_{2}\right)\left[l_{1} l_{2} \dot{\theta}^{2} \sin \left(\theta_{1}-\theta_{2}+\theta_{4}\right)\right] \\
& +m_{2} l_{0}\left[-l_{2} \dot{\theta}^{2} \sin \left(\theta_{2}-\theta_{1}+\theta_{4}-\theta_{3}\right)\right] \\
& -g\left[\left(m_{b}+2 m_{1}+m_{2}\right) l_{2} \sin \left(\theta_{2}-\theta_{1}+\theta+\theta_{p}\right)\right] \\
& +2\left(m_{b}+2 m_{1}+m_{2}\right) l_{2} \dot{l}_{2}\left(\dot{\theta}+\dot{\theta}_{2}-\dot{\theta}_{1}\right)
\end{aligned}
$$




$$
\begin{aligned}
\tau_{3}= & {\left[-l_{1}\left(m_{b}+m_{1}+m_{2}\right) \sin \left(\theta_{2}\right)\right] \ddot{l}_{2}+\left[-l_{1}^{2}\left(m_{b}+m_{1}+m_{2}\right)\right.} \\
& -l_{2}^{2}\left(m_{b}+2 m_{1}+m_{2}\right)-L l_{1}\left(m_{b}+2 m_{1}+2 m_{2}\right) \sin \left(\theta_{3}\right) \\
& +2 l_{1} l_{2}\left(m_{b}+m_{1}+m_{2}\right) \cos \left(\theta_{4}\right) \\
& +\left(m_{1}+m_{2}\right) l_{1}^{2} \cos \left(\theta_{3}+\theta_{1}\right) \\
& +\left(m_{1}+m_{2}\right) l_{1} l_{2} \cos \left(\theta_{4}-\theta_{3}-\theta_{1}\right)-m_{2} l_{1} l_{0} \\
& +\cos \left(\theta_{3}+\theta_{3}-\theta_{4}\right)-m_{2} l_{2} l_{0} \cos \left(\theta_{4}-\theta_{3}+\theta_{2}-\theta_{1}\right) \\
& \left.-L l_{2}\left(m_{b}+2 m_{1}+2 m_{2}\right) \sin \left(\theta_{3}-\theta_{4}\right)\right] \ddot{\theta} \\
& +\left[l_{1}^{2}\left(m_{b}+m_{1}+m_{2}\right)+m_{1} r_{1}^{2}+l_{2}^{2}\left(m_{b}+2 m_{1}+m_{2}\right)\right. \\
& \left.+l_{1} l_{2}\left(m_{b}+m_{1}+m_{2}\right)\right] \ddot{\theta}_{3}+\left[-l_{2}^{2}\left(m_{b}+2 m_{1}+m_{2}\right)\right. \\
& \left.-l_{1} l_{2}\left(m_{b}+m_{1}+m_{2}\right)\right] \ddot{\theta}_{4}+\left[-2\left(m_{b}+2 m_{1}+m_{2}\right) l_{2} \dot{l}_{2}\right. \\
& \left.+\dot{\theta}+\dot{\theta}_{4}-\dot{\theta}_{3}\right)+\left(m_{b}+2 m_{1}+2 m_{2}\right)\left[-L l_{1} \dot{\theta}^{2} \cos \left(\theta_{3}\right)\right. \\
& \left.-L_{2} \dot{\theta}^{2} \cos \left(\theta_{3}-\theta_{4}\right)\right]+\left(m_{b}+m_{1}+m_{2}\right) l_{1} \\
& {\left[-\dot{l}_{2}\left(2 \dot{\theta}_{4}-\dot{\theta}_{3}+\dot{\theta}\right) \cos \left(\theta_{4}\right)\right.} \\
& \left.+l_{2} \dot{\theta}_{4}\left(\dot{\theta}_{4}-\dot{\theta}_{3}+\dot{\theta}\right) \sin \left(\theta_{4}\right)\right] \\
& +\left(m_{1}+m_{2}\right)\left[-l_{1}^{2} \dot{\theta}^{2} \sin \left(\theta_{3}+\theta_{1}\right)\right. \\
& \left.+l_{1} l_{2} \dot{\theta}^{2} \sin \left(\theta_{4}-\theta_{3}-\theta_{1}\right)\right] \\
& +m_{2}\left[l_{1} l_{0} \dot{\theta}^{2} \sin \left(\theta_{3}+\theta_{1}-\theta_{2}\right)\right. \\
& +l_{0} \dot{l}_{2} \dot{\theta}_{\cos } \cos \left(\theta_{4}-\theta_{3}-\theta_{2}+\theta_{1}+2\left(\theta-\theta_{p}\right)\right) \\
& \left.-l_{0} l_{2} \dot{\theta}^{2} \sin \left(\theta_{4}-\theta_{3}+\theta_{2}-\theta_{1}\right)\right] \\
& +g\left[-\left(m_{b}+m_{1}+m_{2}\right) l_{1} \sin \left(\theta_{3}-\theta+\theta_{p}\right)\right. \\
& \left.+\left(m_{b}+2 m_{1}+m_{2}\right) l_{2} \sin \left(\theta_{4}-\theta_{3}+\theta-\theta_{p}\right)\right] \\
& +\dot{m})
\end{aligned}
$$

\begin{tabular}{|c|c|}
\hline Coefficient & Equation \\
\hline$\beta_{10}$ & $\begin{array}{l}\quad\left(\sin (\theta) \sin \left(\theta_{P 1}\right)+\sin (\psi) \cos (\theta) \cos \left(\theta_{P 1}\right)\right) l_{P 1} \\
\quad+\left[-\left(-\sin (\theta) \cos \left(\theta_{P 1}\right)+\sin (\psi) \cos (\theta) \sin \left(\theta_{P 1}\right)\right) \sin \left(\theta_{P 2}\right)\right. \\
\left.\quad+\left(\sin (\theta) \sin \left(\theta_{P 1}\right)+\sin (\psi) \cos (\theta) \cos \left(\theta_{P 1}\right)\right) \cos \left(\theta_{P 2}\right)\right] l_{P 2}\end{array}$ \\
\hline$\beta_{11}$ & $\begin{array}{l}{\left[-\cos (\theta) \sin (\phi) \sin \left(\theta_{P 1}\right)+(\sin (\psi) \sin (\theta) \sin (\phi)+\cos (\psi) \cos (\phi)) \cos \left(\theta_{P 1}\right)\right] l_{P 1}} \\
\quad+\left[-\left(\cos (\theta) \sin (\phi) \cos \left(\theta_{P 1}\right)+(\sin (\psi) \sin (\theta) \sin (\phi)+\cos (\psi) \cos (\phi)) \sin \left(\theta_{P 1}\right)\right) \sin \left(\theta_{P 2}\right)\right. \\
\left.\quad+\left(-\cos (\theta) \sin (\phi) \sin \left(\theta_{P 1}\right)+(\sin (\psi) \sin (\theta) \sin (\phi)+\cos (\psi) \cos (\phi)) \cos \left(\theta_{P 1}\right)\right) \cos \left(\theta_{P 2}\right)\right] l_{P 2}\end{array}$ \\
\hline$\beta_{12}$ & $\begin{array}{l}{\left[\left(-\cos (\theta) \cos (\phi) \sin \left(\theta_{P 1}\right)+\sin (\psi) \sin (\theta) \cos (\phi) \cos \left(\theta_{P 1}\right)-\cos (\psi) \sin (\phi) \cos \left(\theta_{P 1}\right)\right) l_{P 1}\right.} \\
\quad+\left(-\left(\cos (\theta) \cos (\phi) \cos \left(\theta_{P 1}\right)+(\sin (\psi) \sin (\theta) \cos (\phi)-\cos (\psi) \sin (\phi)) \sin \left(\theta_{P 1}\right)\right) \sin \left(\theta_{P 2}\right)\right. \\
\left.\quad+\left(-\cos (\theta) \cos (\phi) \sin \left(\theta_{P 1}\right)+(\sin (\psi) \sin (\theta) \cos (\phi)-\cos (\psi) \sin (\phi)) \cos \left(\theta_{P 1}\right)\right) \cos \left(\theta_{P 1}\right)\right] l_{P 2}\end{array}$ \\
\hline$\beta_{13}$ & $\begin{array}{r}{\left[-\left(-\sin (\theta) \cos \left(\theta_{P 1}\right)+\sin (\psi) \cos (\theta) \sin \left(\theta_{P 1}\right)\right) \sin \left(\theta_{P 2}\right)+\right.} \\
\left.\left(\sin (\theta) \sin \left(\theta_{P 1}\right)+\sin (\psi) \cos (\theta) \cos \left(\theta_{P 1}\right)\right) \cos \left(\theta_{P 2}\right)\right] l_{P 2}\end{array}$ \\
\hline$\beta_{14}$ & $\begin{array}{l}{\left[-\left(\cos (\theta) \sin (\phi) \cos \left(\theta_{P 1}\right)+(\sin (\psi) \sin (\theta) \sin (\phi)+\cos (\psi) \cos (\phi)) \sin \left(\theta_{P 1}\right)\right) \sin \left(\theta_{P 2}\right)\right.} \\
\left.\quad+\left(-\cos (\theta) \sin (\phi) \sin \left(\theta_{P 1}\right)+(\sin (\psi) \sin (\theta) \sin (\phi)+\cos (\psi) \cos (\phi)) \cos \left(\theta_{P 1}\right)\right) \cos \left(\theta_{P 2}\right)\right] l_{P 2}\end{array}$ \\
\hline$\beta_{15}$ & $\begin{array}{l}{\left[-\left(\cos (\theta) \cos (\phi) \cos \left(\theta_{P 1}\right)+(\sin (\psi) \sin (\theta) \cos (\phi)-\cos (\psi) \sin (\phi)) \sin \left(\theta_{P 1}\right)\right) \sin \left(\theta_{P 2}\right)\right.} \\
\left.\quad+\left(-\cos (\theta) \cos (\phi) \sin \left(\theta_{P 1}\right)+(\sin (\psi) \sin (\theta) \cos (\phi)-\cos (\psi) \sin (\phi)) \cos \left(\theta_{P 1}\right)\right) \cos \left(\theta_{P 2}\right)\right] l_{P 2}\end{array}$ \\
\hline
\end{tabular}

$$
\begin{aligned}
\tau_{4}= & {\left[\left(m_{b}+2 m_{1}+m_{2}\right) l_{2}^{2}+\left(m_{b}+2 m_{1}+m_{2}\right)\right.} \\
& L l_{2} \sin \left(\theta_{3}-\theta_{4}\right) \\
& +\left(m_{b}+m_{1}+m_{2}\right) l_{1} l_{2} \cos \left(\theta_{4}\right)-\left(m_{1}+m_{2}\right) l_{1} l_{2} \\
& \left.\cos \left(\theta_{4}-\theta_{3}-\theta_{1}\right)+m_{2} l_{0} l_{2} \cos \left(\theta_{4}-\theta_{3}+\theta_{2}-\theta_{1}\right)\right] \ddot{\theta} \\
& +\left[-l_{2}^{2}\left(m_{b}+2 m_{1}+m_{2}\right)-l_{1} l_{2}\left(m_{b}+m_{1}+m_{2}\right)\right. \\
& \left.\cos \left(\theta_{4}\right)\right] \ddot{\theta}_{1}+\left[l_{2}^{2}\left(m_{b}+2 m_{1}+m_{2}\right)\right] \ddot{\theta}_{4} \\
& +2\left(m_{b}+2 m_{1}+m_{2}\right) l_{2} \dot{l}_{2}\left(\dot{\theta}+\dot{\theta}_{4}-\dot{\theta}_{3}\right) \\
& +\left(m_{b}+2 m_{1}+m_{2}\right) L \dot{l}_{2} \dot{\theta} \sin \left(\theta_{3}-\theta_{4}\right) \\
& +\left(m_{b}+2 m_{1}+2 m_{2}\right) L l_{2} \dot{\theta} \cos \left(\theta_{3}-\theta_{4}\right) \\
& +\left(m_{b}+m_{1}+m_{2}\right) l_{1} l_{2}\left(\dot{\theta}_{3}-\dot{\theta}\right)^{2} \sin \left(\theta_{4}\right) \\
& +\left(m_{b}+2 m_{1}+2 m_{2}\right) L\left(l_{2} \dot{\theta}\left(\dot{\theta}+\dot{\theta}_{4}-\dot{\theta}_{3}\right)\right. \\
& \left.\cos \left(\theta_{3}-\theta_{4}\right)-\dot{l}_{2} \dot{\theta} \sin \left(\theta_{3}-\theta_{4}\right)\right) \\
& +\left(m_{1}+m_{2}\right)\left[-l_{1} l_{2} \dot{\theta}^{2} \sin \left(\theta_{4}-\theta_{3}-\theta_{1}\right)\right] \\
& +m_{2} l_{0}\left[\dot{l}_{2} \dot{\theta} \cos \left(\theta_{4}-\theta_{3}+\theta_{2}-\theta_{1}\right)\right. \\
& -\dot{l}_{2} \dot{\theta} \cos \left(\theta_{4}-\theta_{3}-\theta_{2}+\theta_{1}+2\left(\theta-\theta_{p}\right)\right) \\
& \left.+l_{2} \dot{\theta}^{2} \sin \left(\theta_{4}-\theta_{3}+\theta_{2}-\theta_{1}\right)\right] \\
& -g\left[\left(m_{b}+2 m_{1}+m_{2}\right) l_{2} \sin \left(\theta_{4}-\theta_{3}+\theta-\theta_{p}\right)\right] \\
& \left.+\theta{ }_{3}\right)
\end{aligned}
$$

\section{Appendix B}

Coefficients used in equations to determine the required efforts at the joints of the two-DOF planar robot 


\section{Nomenclature}

$L \quad$ Half the distance between the hip joints

$m_{\mathrm{b}} \quad$ Body mass

$r_{\mathrm{b}} \quad$ Body radius of gyration

$k \quad$ Spring stiffness

$b \quad$ Damping coefficient

$l_{\mathrm{o}} \quad$ Free leg length (zero spring force)

$l_{1} \quad$ Link 1 length

$l_{2} \quad$ Link 2 length

$m_{1} \quad$ Mass of link 1

$m_{2} \quad$ Mass of link 2

$\theta_{\mathrm{p}} \quad$ Inclination of plane w.r.to horizontal

$\theta \quad$ Body angle w.r.to inclined plane

$\theta_{1} \quad$ Angle of rotation of back leg (link 1)

$\theta_{2} \quad$ Angle of rotation of back leg (link 2)

$\theta_{3} \quad$ Angle of rotation of front leg (link 1)

$\theta_{4} \quad$ Angle of rotation of front leg (link 2)

$\tau_{1} \quad$ Torque applied at back leg (link 1)

$\tau_{2} \quad$ Torque applied at back leg (link 2)

$\tau_{3} \quad$ Torque applied at front leg (link 1)

$\tau_{4} \quad$ Torque applied at front leg (link 2)

$\theta_{P 1} \quad$ Angle of rotation of frame $\left\{P_{1}\right\}$ of two DOF planar robot

$\theta_{P 2} \quad$ Angle of rotation of frame $\left\{P_{2}\right\}$ of two DOF planar robot

$\phi, \theta, \psi \quad Z-Y-X$ Euler angles

\section{References}

[1] Chen X, Gao F, Qi C and Zhao X 2013 Spring parameters design to increase the loading capability of a hydraulic quadruped robot. In: Proceeding of the International conference on Advanced Mechatronics Systems, Luoyang, China, pp. 535-540

[2] Spröwitz A, Tuleu A, Vespignani M, Ajallooeian M, Badri E and Ijspeert A 2013 Towards dynamic trot gait locomotion: Design, control, and experiments with Cheetah-cub, a compliant quadruped robot. The International Journal of Robotics Research, 32(8): 932-950

[3] Semini C, Tsagarakis N, Guglielmino E, Focchi M, Cannella F and Caldwell D 2011 Design of HyQ - a hydraulically and electrically actuated quadruped robot. Proceedings of the Institution of Mechanical Engineers, Part I: Journal of Systems and Control Engineering. 225(6): 831-849

[4] Byl K 2008 Metastable Legged-Robot Locomotion. Ph.D. Thesis, Massachusetts Institute of Technology

[5] Shkolnik A, Levashov M, Manchester I and Tedrake R 2011 Bounding on rough terrain with the LittleDog robot. The International Journal of Robotics Research. 30: 192-215

[6] Fukuoka Y and Kimura H 2009 Dynamic locomotion of a biomorphic quadruped 'Tekken' robot using various gaits: walk, trot, free-gait and bound. Applied Bionics and Biomechanics 6: 63-71

[7] Kimura H and Fukuoka Y 2004 Biologically inspired adaptive dynamic walking in outdoor environment using a selfcontained quadruped robot:'Tekken2'. In: Proceeding of
IEEE/RSJ International Conference on Intelligent Robots and Systems, (IROS 2004). pp. 986-991

[8] Lee Y-J and Hirose S 2000 Three-Legged Walking for Fault Tolerant Locomotion of a Quadruped Robot with Demining Mission. In: Proceedings of the 2000 IEEE/RSJ International Conference on intelligent Robots and Systems, pp. 973-978

[9] Yang J-M 2002 Fault-tolerant gaits of quadruped robots for locked joint failures. IEEE Transactions on Systems, Man and Cybernetics-Part C, 32(4): 507-516

[10] Yang J-M 2003 Crab walking of quadruped robots with a locked joint failure. Advanced Robotics, 17(9): 863-878

[11] Yang J-M 2008 Two-phase discontinuous gaits for quadruped walking machines with a failed leg. Robotics and Autonomous Systems, 56(9): 728-737

[12] Krishnan V L, Pathak P M, Jain S C and Samantaray A K 2011 Reconfiguration of four-legged walking robot for actuator faults. Proceedings of the Institution of Mechanical Engineers, Part I: Journal of Systems and Control Engineering, 225: 1-16

[13] Gor M, Pathak P, Samantaray A, Yang J and Kwak S 2013 Dynamic Modeling and Simulation of Compliant Legged Quadruped Robot. In: Proceeding of the 1st International and 16th National Conference on Machines and Mechanisms (iNaCoMM2013), IIT Roorkee, India, Dec 18-20 2013. pp. 7-16

[14] Gor M, Pathak P, Samantaray A, Yang J and Kwak S 2015 Control oriented model-based simulation and experimental studies on a compliant legged quadruped robot. Robotics and Autonomous System 72: 217-234

[15] Ganesh Kumar K and Pathak P 2013 Dynamic modelling and simulation of a four legged jumping robot with compliant legs. Robotics and Autonomous Systems 61(2013): 221-228

[16] Estremera J and Waldron K 2008 Thrust control, stabilization and energetics of a quadruped running robot. International Journal of Robotics Research 27: 1135-1151

[17] Xiuli Z, Haojun Z, Xu G, Zhifeng C and Liyao Z 2005 A biological inspired quadruped robot: structure and control. IEEE International Conference on Robotics and Biomimetics 387-392

[18] Raibert M, Chepponis M and Brown B 1986 Running on four legs as though they were one. IEEE Journal of Robotics and Automation, RA-2. 70-82

[19] Hodoshima R, Doi T, Okamoto T and Mori J 2004 Development of TITAN XI: a Quadruped Walking Robot to Work on Slopes. In: Proceeding of IEEE/RSJ International Conference on Intelligent Robots and Systems, September 28-October 2, 2004, Sendal, Japan. pp. 792-797

[20] Tanaka T and Hirose S 2008 Development of leg-wheel hybrid quadruped airhopper - design of powerful lightweight leg with wheel. In: Proceedings of the IEEE/RSJ International Conference on Intelligent Robots and Systems (IROS), 2008, pp. 3890-3895

[21] Poulakakis I, Smith J and Buehler M 2005 Modeling and experiments of untethered quadrupedal running with a bounding gait: The scout II robot. International Journal of Robotics Research 24: 239-256

[22] Kurfess T 2004 Robotics and Automation Handbook. Boca Raton: CRC Press

[23] http://www.maxonmotor.in/

[24] http://www.invensense.com/mems/gyro/mpu6050.html

[25] http://www.ptiphoenix.com/products/

[26] Craig, J J 2005 Introduction to Robotics Mechanics and Control, $3^{\text {rd }}$ ed. USA: Pearson Education 